Ceci n'est pas une guerre (mais ça y ressemble) : entre doctrine et sémantique, comment aborder la question de la guerre préhistorique?

This is not a war (though it looks like one): between doctrine and semantics, how to address the issue of prehistoric war?

\title{
Bruno Boulestin
}

\section{OpenEdition}

Journals

Édition électronique

URL : http://journals.openedition.org/paleo/5073

DOI : $10.4000 /$ paleo.5073

ISSN : 2101-0420

Éditeur

SAMRA

Édition imprimée

Date de publication : 1 septembre 2020

Pagination : $36-56$

ISSN : $1145-3370$

Référence électronique

Bruno Boulestin, «Ceci n'est pas une guerre (mais ça y ressemble) : entre doctrine et sémantique, comment aborder la question de la guerre préhistorique? », PALEO [En ligne], 30-2 | 2020, mis en ligne le 30 septembre 2020, consulté le 26 novembre 2020. URL : http://journals.openedition.org/paleo/ 5073 ; DOI : https://doi.org/10.4000/paleo.5073

\section{(9) $\odot \Theta \Theta$}

PALEO est mis à disposition selon les termes de la licence Creative Commons Attribution - Pas d'Utilisation Commerciale - Pas de Modification 4.0 International. 
Bruno Boulestina,

a. Université de Bordeaux, UMR 5199-PACEA ; Allée Geoffroy Saint-Hilaire, CS 50023 - Bâtiment B8, FR-33615 PESSAC Cedex -

bruno.boulestin@u-bordeaux.fr.
Les discussions sur la guerre préhistorique se heurtent à deux grands problèmes. Le premier relève de la doctrine, la réflexion étant dès le départ faussée par les préjugés que chacun peut avoir à propos de la possibilité ou non de son existence. Le second est de nature sémantique : qu'appelle-t-on guerre? Du point de vue de la doctrine, on peut distinguer deux courants épistémologiques: certains considèrent que la guerre existe dans les sociétés les plus simples, y compris celles du Paléolithique, tandis que pour d'autres la guerre est absente dans ces sociétés et n'a émergé que tardivement dans l'histoire de l'humanité. Ces deux courants reflètent fondamentalement deux vues opposées sur la nature humaine, et les présupposés qui leur sont attachés constituent des biais importants. Du point de vue sémantique, malgré la quantité d'écrits sur la guerre, il n'en existe aucune définition qui soit générale et consensuelle. Parmi tous les éléments qui ont été utilisés pour la caractériser, on ne peut en dégager que trois qui soient réellement fondamentaux: le recours à la violence armée, la nature intergroupe du phénomène, en gardant au concept de groupe un sens très général, et le fait qu'il s'agit d'un état d'hostilité. S'ajoute, en langue anglaise, la difficulté de différencier guerre et feud, due à ce que feud décrit en réalité deux formes de vengeance, dont une est assimilable à de la guerre. Finalement, considérées objectivement, les données n'excluent en rien que les chasseurs-cueilleurs du Paléolithique aient pu faire la guerre. En revanche, bien des raisons rendent la guerre préhistorique difficile à démontrer. La guerre doit donc faire partie des hypothèses invoquées pour expliquer les cas paléolithiques de violence armée. Mais, avant tout, la question de la guerre préhistorique doit être abordée de manière objective, en s'affranchissant de tout a priori.
PALE0 30 | t. 2

JUILLET 2020

PAGES 36 À 56
MOTS-CLÉS Guerre, guerre primitive, guerre préhistorique, faide. 


\section{This is not a war (though it looks like one):}

between doctrine and semantics, how to address

\section{the issue of prehistoric war?}

Discussions on prehistoric war usually face two major issues. The first one pertains to doctrine, since the reflection is distorted from the very beginning by everyone's prejudices regarding the possibility or not that such war actually existed. The second issue belongs to the semantic field: what do we call war? From a doctrinal point of view, one can identify two main epistemological trends: some consider that war is a very ancient phenomenon, dating back at least to the Palaeolithic, and that it can also be observed in simple societies from the recent past, while for others war appears quite late in the history of mankind and does not exist in simple societies from the recent past. Fundamentally, these two trends mirror two opposite visions of the human nature, and the connected presuppositions inherently bias the discussions on prehistoric war. In regard to the semantics, despite the number of works dealing with war, it has never been defined, either generally or consensually. In truth, among the elements used to define war, only three are fundamental: the use of armed violence, the intergroup nature of the phenomenon (keeping a very general meaning to the concept of "group"), and the fact that war is a state of hostility. In addition, in the English language, there is the difficulty of distinguishing between war and feud, due to the fact that "feud" actually describes two different forms of revenge, one of which can be likened to war. Finally, if we objectively consider the data, there is no reason why the Palaeolithic hunter-gatherers could not have been at war, even if, on another hand, prehistoric war is actually difficult to prove. The hypothesis of war must be among those put forward to explain the examples of Palaeolithic armed violence, since there is no reason why it should be rejected. Before all though, addressing the issue of prehistoric warfare must be done with objectivity and disregarding any a priori.

KEY-WORDS Prehistoric war, primitive war, war, warfare, feud.

\section{INTRODUCTION}

À peu près tout ceux qui travaillent sur la violence s'accordent à dire qu'elle existe depuis des temps extrêmement anciens. Mais en ce qui concerne la guerre, il en va différemment. Certains parmi eux pensent également qu'elle est aussi vieille que l'humanité, d'autres non. Pour les seconds, ipso facto il y a une frontière temporelle qui sépare une période de l'histoire humaine durant laquelle la guerre n'existait pas, d'une autre (la nôtre) où elle existe. Cette frontière n'est pas tant un seuil au-delà duquel, à leurs yeux, la guerre ne serait plus démontrable, qu'une limite que l'on peut qualifier de conceptuelle, qui en pratique se manifeste sous deux formes. D'un côté, il y a ceux qui n'admettent tout simplement pas l'existence de la guerre avant une certaine époque ; elle serait donc apparue tardivement. De l'autre, il y a les auteurs qui ont une position plus nuancée, intermédiaire entre la précédente et l'idée que la guerre a toujours existé : tout en reconnaissant la présence de quelque chose qui s'apparente à la guerre au-delà de la frontière, ils ont des réticences à utiliser le terme, doutant qu'il soit réellement adapté. En France, ces deux positions sont par exemple représentées respectivement par $M$. Patou-Mathis (2016) et J. Guilaine (2011). En pratique, selon les chercheurs cette frontière se situe généralement quelque part entre la fin des temps glaciaires et la néolithisation, mais certains la déplacent même jusqu'au début des âges des métaux.

Apparaissent ici les deux problèmes auxquels se heurte, et cela avant même d'avoir débuté, toute réflexion sur la guerre préhistorique. Le premier est un problème de doctrine : l'approche différera en fonction des convictions, selon qu'il sera considéré a priori que la guerre a tout simplement pu ou non exister au Paléolithique. Le second est un problème de sémantique : qu'appelle-t-on « guerre »? Et, plus encore, le terme a-t-il seulement un sens dans certaines sociétés ? Ces deux problèmes, qui sont en partie reliés, ne sont en fait pas propres à la question de la guerre préhistorique. On verra notamment plus loin que cette question s'inscrivant dans une perspective évolutionniste, elle a tout naturellement son pendant en ethnologie, la frontière supposée entre guerre et nonguerre n'y étant plus temporelle, mais sociale. Plus largement, l'essai tout récent de C. Hass (2019) montre bien que même en Histoire contemporaine, là où l'existence de la guerre ne fait aucun doute et où le concept paraît correctement défini, les problèmes de doctrine et de sémantique sont loin d'être absents.

Avant d'évoquer tour à tour ces deux aspects plus en détail, un avertissement s'impose d'emblée. Il faut naturellement essayer de leur trouver des réponses, c'està-dire d'une part réfléchir à la manière dont on peut aborder la question de la guerre préhistorique en ayant la démarche la plus objective possible, d'autre part tenter de comprendre ce qui, d'une façon générale, peut le mieux caractériser la guerre. Mais il s'agit plus de donner une position que d'apporter les solutions. La guerre est en effet une affaire si complexe que l'on tenterait en vain d'en embrasser toutes les dimensions, et elle a donné lieu à une littérature si considérable qu'il est devenu aujourd'hui extrêmement difficile, sinon impossible de la maîtriser totalement. Son domaine d'étude est si vaste, qui recouvre des disciplines aussi variées que l'anthropologie, l'ar- 
chéologie, la sociologie, l'ethnologie, l'histoire, les sciences politiques, la psychologie cognitive, la philosophie, etc., qu'il porte son propre nom, "polémologie » (pour les anglophones polemology, équivalent des war studies), nom que l'on doit à G. Bouthoul (1951), qui fut l'un de ses plus grands spécialistes. Rien qu'en s'en tenant à l'anthropologie sociale, on trouve de multiples écoles de pensée (écologie culturelle, matérialisme culturel, structuralisme, sociobiologie, etc.) et autant de théories qui donnent lieu à de multiples controverses. Pour se rendre compte du nombre ahurissant de travaux ne serait-ce que dans cette seule discipline, il suffit de citer le cas emblématique des Yanomami : depuis la publication de N. Chagnon (1968), il y a cinquante ans, on en compte sans doute plus d'une centaine rien que sur cette société, illustrant toutes les interprétations possibles (résumées dans Ferguson 2001). On se recentrera donc ici sur les contributions directement utiles aux propos, sans prétention d'exhaustivité. Des synthèses et bibliographies complémentaires sur l'histoire de la recherche et les différents courants de pensée pourront être trouvées dans, entre autres, Ferguson et Farragher (1988), Haas (1990), Dennen (1995), Otterbein (1999, 2004), Fry (2007, 2013), Gat (2006, 2017), Roscoe (2017). Pour les ouvrages récents en français, outre celui de J. Guilaine et J. Zammit (2001), mentionnons la série sur la sociologie de la guerre, publiée sous la direction de J. Baechler depuis 2014 (notamment Baechler 2019). Mais d'une manière générale, l'intérêt pour la guerre, en particulier dans les sociétés non étatiques, a infiniment plus marqué le monde anglosaxon que francophone, lequel se signale par le peu de recherches sur cette question, tant sur le versant archéologique qu'ethnologique.

\section{1 | ÉPISTÉMOLOGIE DE LA GUERRE : BRÈVE VISION D'ENSEMBLE}

\section{1 | Deux grands courants épistémologiques}

Dans l'immense domaine des études auxquelles la guerre a donné lieu au cours des dernières décennies, il est possible de distinguer deux grands courants épistémologiques, dont, suite à K. Otterbein (1997), les partisans sont qualifiés par les Anglo-Saxons respectivement de faucons et de colombes (hawks et doves, en référence à la guerre anglo-américaine de 1812). Plus classiquement, ces courants se réfèrent aux positions adoptées d'un côté par Thomas Hobbes et de l'autre par Jean-Jacques Rousseau : à l'état de nature, pour le premier c'est «la guerre de tous contre tous», pour le second l'homme est un «bon sauvage ».

Les faucons sont donc les représentants du courant que l'on peut qualifier de manière générale d'hobbesien. L'homme est vu par eux comme naturellement agressif, et il n'a été domestiqué que peu à peu, par la civilisation. De ce fait, la guerre est dans la nature humaine : c'est un phénomène plus ou moins universel qui, s'il n'a toujours existé, possède au moins une très grande ancienneté. Parmi les représentants de cette conception, on peut citer N. Chagnon (1968), J. M. G. van der Dennen (1995), L. H. Keeley (1996), S. A. LeBlanc (LeBlanc et Register 2003), A. Gat $(2006,2017)$, ou en France P. Clastres (1980 - p. 171 sq., 1997) ou P. Descola et M. Izard (1991).
Tous les chercheurs n'adoptent toutefois pas la vision hobbesienne stricte d'une humanité qui aurait connu « la guerre de tous contre tous », et l'on trouve plusieurs tendances au sein de ce courant. K. Otterbein aux ÉtatsUnis ou J. Guilaine et J. Zammit en France, bien qu'ils puissent être considérés comme étant dans le camp des faucons dans la mesure où ils admettent l'existence d'au moins une forme de guerre dès le Paléolithique, ont une position mesurée. Le premier préconise une attitude intermédiaire et se définit lui-même comme étant à la fois dans le camp des faucons et dans celui des colombes (Otterbein 1997, 2004 - p. XIII). Pour les seconds, la violence intergroupe a peu ou prou toujours existé, mais en fonction de la structure des sociétés les affrontements se traduisent diversement. Selon eux, plutôt que d'envisager la guerre comme une pratique étroitement liée à l'apparition de certaines formes de sociétés, il semble plus pertinent de penser l'émergence de ce comportement sur une perspective de temps long, avec, sans doute, une véritable « préhistoire de la guerre » (Guilaine et Zammit 2001). À un degré supérieur, certains, comme Keeley, proposent de faire remonter l'origine de la guerre aux temps les plus reculés de l'Histoire humaine. Il s'agirait à tout le moins d'une constante de l'écrasante majorité des sociétés humaines, à travers le temps et l'espace, et les guerres dites « primitives » auraient été fréquentes et le plus souvent autant destructrices et violentes que les guerres modernes. Enfin, d'autres encore estiment que l'origine de la violence collective (sinon de la guerre) serait à rechercher dans les comportements violents intercommunautaires chez certains animaux, en particulier les singes (il faudrait donc remonter au-delà de la séparation des différents Homininés). C'est une position notamment défendue par les socioécologistes, comme R. Wrangham (Wrangham 1999 ; Wrangham et Glowacki 2012) ou A. Gat $(2006,2017)$. Dans tous les cas et quelle que soit la tendance à laquelle ils se rattachent, les représentants de ce courant estiment que les chasseurs-cueilleurs du passé récent documentés par l'ethnographie pratiquaient également la guerre.

Les colombes sont les représentants du courant rousseauiste, selon lequel l'homme est naturellement pacifique et la guerre n'aurait émergé que tardivement dans l'histoire de l'humanité, à partir d'un certain niveau de développement, qui toutefois varie selon les auteurs. Dans ce cadre, d'une manière générale les sociétés " primitives » ne connaissent pas la guerre, ou en tout cas pas la "vraie guerre», mais tout au plus une guerre qualifiée elle aussi de « primitive » ou de «proto-guerre », une façon de dire que ce n'en est pas vraiment une. Parmi les partisans de ce courant, on peut mentionner B. Malinowski (1941), Q. Wright (1942), H. H. Turney-High (1949), R. C. Kelly (2000), D. P. Fry (2007), R. B. Ferguson (2004, 2008, 2018), ou en France M. Escalon de Fonton (1964), M. PatouMathis $(2013,2016)$ ou J.-P. Demoule (2017).

Comme pour le premier courant, celui-ci est marqué par plusieurs tendances, mais on peut simplement en distinguer deux grandes. La première, qui est aussi la plus ancienne, est représentée par exemple par Wright, TurneyHigh ou Escalon de Fonton. Au sein de cette tendance, les sociétés non étatiques, qu'elles soient anciennes ou modernes, se voient dénier toute dimension guerrière: elles n'ont pas connu la guerre ou ne la connaissent pas. Dépourvues des institutions sociopolitiques et économiques qui fondent les États, ces sociétés n'ont pas 
pu développer de véritables conflits armés. Tout au plus ont-elles mis en place des manifestations de piètre ampleur destinées à régler à moindres frais les querelles pouvant exister entre elles, de types expéditions ou raids peu organisés, avec une faible mobilisation et conduisant à des affrontements peu mortels. Mais en aucun cas il ne peut s'agir de véritables guerres, lesquelles supposeraient une « véritable armée », avec tout ce que cette conception suppose (tactique, stratégie, logistique, entraînement, discipline, hiérarchie...). Pour les tenants de cette tendance, en ethnologie la question de la guerre dans les sociétés traditionnelles ne se pose même pas. Par définition, les combats qui peuvent y être observés ne relèvent pas de la vraie guerre, puisqu'il n'y existe pas de véritables armées. En archéologie, cette question n'a pas plus de sens qu'en ethnologie : la guerre apparaîtrait avec les premières grandes civilisations (l'histoire de la guerre commence à Sumer, pour paraphraser le titre du célèbre ouvrage de S. N. Kramer). Qu'il y ait ou non des traces archéologiques antérieures n'a de toute manière que peu d'importance : les affrontements qui ont pu exister avant ne relèvent pas de la vraie guerre. Cette première tendance, plutôt extrême, a toutefois cédé le pas devant l'évidence et n'est plus strictement représentée aujourd'hui, la position étant devenue intenable face à l'accumulation des données ethnographiques ou archéologiques au cours des dernières décennies. Néanmoins, elle se retrouve sous une forme atténuée chez ceux qui continuent à relier la « vraie » guerre à un certain degré d'évolution des techniques.

Ceux, très majoritaires, qui se rattachent à la seconde grande tendance chez les colombes, considèrent que la guerre émerge avec la sédentarisation des sociétés et leur complexification (au sens du développement des inégalités socioéconomiques et des hiérarchies qui en découlent, voir infra) et qu'elle s'intensifie avec l'économie de production. En ce qui concerne l'ethnologie, cela s'accompagne d'une méfiance certaine vis-à-vis des travaux qui se fondent sur les résultats des enquêtes ethnographiques, voire par leur rejet, notamment quand ces enquêtes portent sur les chasseurs-cueilleurs dits simples (c'est-à-dire égalitaires, mobiles et non stockeurs). La raison avancée est que les données ethnographiques ne peuvent pas fournir des éléments de compréhension des conflits préhistoriques, car partout les contacts avec les civilisations étatiques, notamment européennes, ont modifié le comportement des sociétés. Les travaux de R. B. Ferguson (Ferguson 1992, 2004 ; Ferguson et Whitehead 1992) sont représentatifs de cette attitude. Ainsi, écrit-il que " partout dans le monde, ce qu'on appelle la guerre primitive, ou indigène, a généralement été transformée, fréquemment intensifiée et parfois précipitée par le contact avec les Occidentaux. [...] La guerre indigène décrite dans les siècles récents ne peut donc être considérée comme typique de la guerre préhistorique »(Ferguson 2004 - p. 52). Cette théorie, qualifiée de contact paradox par Gat (2006 passim, 2015, 2017 - p. 6-15 et passim), qui par ailleurs la réfute, est même étendue par Ferguson (2018) aux chimpanzés, puisqu'il avance que leur violence peut être liée à des circonstances spécifiques résultant de perturbations de leur vie par le contact avec les humains. En ce qui concerne l'archéologie, le rejet de la guerre paléolithique se fait sur la base de deux types d'arguments : d'une part il y a des raisons pour qu'elle n'existe pas, d'autre part on n'en a pas de preuve archéologique. Le premier renvoie, en miroir, à l'idée que la guerre apparaît avec la complexité sociale, les chasseurs-cueilleurs du Paléolithique étant tenus comme ayant été simples. Quant au second argument, il est facile à comprendre, et on le trouve résumé encore une fois par Ferguson (2004 - p. 54) : " si la guerre était courante dans les temps anciens préhistoriques, les abondants vestiges archéologiques devraient en contenir les traces. Or, il n'en est rien ». Ce qui est plus subtil, c'est que cet argument vient en réalité en complément du rejet des données ethnographiques: puisque ces dernières sont biaisées, seules les données archéologiques peuvent permettre d'identifier les conflits anciens. Or si ces données font défaut, c'est que les conflits eux-mêmes n'existaient pas. Dans ce cadre conceptuel, en pratique la plus ancienne manifestation tangible d'une guerre communément admise est celle de Jebel Sahaba en Nubie égyptienne (Wendorf 1968), pour des chasseurs-cueilleurs ayant vécu autour de 12000 ans avant notre ère. Et encore, Patou-Mathis (2013 - p. 150151, 2016 - p. 28) admet-elle la violence collective à propos de ce cas, mais en émettant des doutes sur sa nature guerrière, et Ferguson (2018) met-il en avant que ces chasseurs-cueilleurs étaient déjà sans doute sédentaires ou semi-sédentaires. Ailleurs, la guerre aurait émergé à différentes époques selon les régions, mais quoi qu'il en soit au plus tôt au Mésolithique, entendu comme la période durant laquelle, au moins dans certains endroits, des chasseurs-cueilleurs simples seraient devenus complexes.

\section{2 | Quelques réflexions générales}

Quelle attitude adopter vis-à-vis de ces deux grands courants épistémologiques? Avant d'apporter des éléments de réponse à cette question, il faut s'arrêter sur une autre, celle de la définition de la guerre, si ce n'est pour lui trouver une solution définitive, au moins pour en donner les tenants et les aboutissants. Néanmoins, on peut déjà faire quelques remarques de portée générale. La première, c'est que dans tous les cas, la perception de la guerre en archéologie et celle en ethnohistoire sont le strict reflet l'une de l'autre. Il ne peut en être autrement. Pour les faucons, c'est une question de cohérence: soutenir que la guerre existe depuis la nuit des temps implique de considérer qu'elle est présente à toutes les époques et dans tous les types de sociétés. Quant aux colombes, celles qui nient l'existence de la guerre avant la naissance de l'État doivent obligatoirement la rejeter aussi dans les sociétés non étatiques historiques, tandis que celles qui considèrent que les chasseurs-cueilleurs simples préhistoriques ne connaissaient pas la guerre sont conduits à récuser les sources ethnologiques qui font état de guerres chez les mêmes chasseurs-cueilleurs du passé récent.

La deuxième remarque vient compléter la précédente. Il est frappant de constater que les deux tendances présentes chez les colombes placent l'origine de la guerre à deux moments différents, mais qui ont en commun d'être couramment considérés comme les deux grandes charnières dans l'évolution des sociétés humaines. C'est particulièrement explicite si l'on se réfère aux classifications néo-évolutionnistes américaines, qui distinguent globalement trois grands stades de cette évolution : les sociétés simples, les sociétés complexes 
pré-étatiques et les États. Si les premières se définissent surtout par l'absence des caractéristiques des suivantes, elles correspondent en pratique aux chasseurs-cueilleurs égalitaires, c'est-à-dire à peu près aux sociétés « achrématiques » (monde I) de Testart (2005)'. Les deuxièmes (prestate sedentary societies ou middle-range societies) sont sédentaires, hiérarchisées, stockent et connaissent la richesse. Concrètement, elles regroupent tout ce qui se trouve entre la « bande » et l'État, c'est-àdire les «tribus» et les « chefferies», dont celles de chasseurs-cueilleurs complexes (sur ces notions, assez mal définies, et sur les difficultés que posent les classifications des middle-range societies, voir par exemple Feinman et Neitzel 1984). Il y a donc bien quelque part l'idée chez les colombes qu'il faut lier la naissance de la guerre à un profond changement dans l'organisation des sociétés, voire la justifier par une sorte de rupture évolutive de ce point de vue. Pour les auteurs du milieu du siècle dernier, cette rupture était l'apparition de l'État. Mais quand la position n'a plus été tenable, c'est l'autre rupture, entre simplicité et complexité, qui a été choisie comme position de repli. On peut y voir une sorte de dernier bastion auquel s'accrochent les colombes et qu'elles font tout pour défendre (Allen 2014). Sans doute parce qu'admettre que la guerre peut exister dans les sociétés simples, ce serait en quelque sorte faire tomber la dernière barrière, celle au-delà de laquelle il ne serait plus possible de relier, au moins de façon aussi évidente, guerre et évolution sociale. Pourquoi dès lors la guerre ne serait-elle pas aussi ancienne, sinon que l'humanité, au moins qu'Homo sapiens?

Ces remarques débouchent sur une réflexion plus large. Quand on regarde de près, la raison du désaccord entre les partisans des deux courants n'est pas seulement à rechercher dans la difficulté d'interprétation des données empiriques, mais tout autant dans leurs présupposés philosophiques sur ce que sont censées montrer l'ethnologie et l'archéologie sur la nature humaine. Plusieurs auteurs l'ont d'ailleurs noté, par exemple Jones et Allen (2014 - p. 354) : "Scholars seem to rarely approach the topic without predisposition, and frequently assemble and interpret empirical evidence with certain outcomes in mind. » En effet, la question de la guerre préhistorique renvoie à celle des racines mêmes du phénomène et de sa trajectoire, et selon l'opinion que l'on aura à propos de la guerre « primitive » et de son origine, on expliquera différemment la guerre contemporaine. Mais, plus encore, l'interrogation sur quand et comment cela a-t-il démarré fait écho à une autre préoccupation essentielle : pouvonsnous en finir, et comment ? C'est donc bien sur la nature humaine que porte le fond du débat : la guerre est-elle consubstantielle à l'Homme, inscrite dans ses gènes, ou bien est-elle culturelle et choisit-il de la faire ? Ce n'est jamais qu'un aspect parmi d'autres de l'éternel conflit entre nature et culture, entre biologique et social. Faucons et colombes, ce sont avant tout deux vues opposées de l'humanité. Il n'est cependant pas compliqué de compren-

[1] Dans les classifications néo-évolutionnistes, la différence entre sociétés se fonde en premier lieu sur le politique, tandis que chez Testart la différence entre mondes est basée sur l'économie. L'équivalence entre existence de la richesse et présence de hiérarchie étant presque stricte, on peut pratiquement assimiler sociétés simples et monde I (qui regroupe essentiellement les chasseurs-cueilleurs nomades, mais aussi quelques horticulteurs). Par contre, les États se rencontrent à la fois dans les mondes II et III de Testart, le passage entre les deux se faisant avec l'apparition de la propriété fundiaire. dre que tous ces présupposés, qui rendent la frontière entre science et idéologie poreuse, faussent en grande partie les discussions sur l'existence de la guerre au Paléolithique, dans un sens ou un autre.

\section{2 | LA DIFFICILE QUESTION DE LA DÉFINITION DE LA GUERRE}

\section{1 | État de la question}

La première difficulté rencontrée pour discuter la guerre, qu'elle soit ou non préhistorique, est tout simplement de savoir ce qu'elle est. Aussi étonnant que cela puisse paraître, malgré l'ancienneté des débats sur le sujet et les sommes considérables dépensées en encre pour des publications, il n'existe aucune définition de la guerre qui soit générale et consensuelle. Et ce n'est pas qu'il s'agirait de choisir entre quelques-unes : on en recense probablement plus d'une centaine. Même l'OMS a renoncé et tend à utiliser préférentiellement l'expression « conflit armé » à « guerre » étant donné la complexité à définir cette dernière (Zwi et al. 2002 - p. 216). Elle n'est pas la seule à avoir glissé la difficulté sous le tapis, nombre de publications sur la guerre pré ou protohistorique (en particulier françaises) réussissant à ne cerner leur sujet d'étude à aucun moment, ce qui est tout de même paradoxal. La définition de la guerre semble donc insaisissable, pour reprendre une formulation de N. Werly (2002) à propos de celle de la paix - que la constatation soit la même pour l'une et l'autre n'a d'ailleurs rien d'étonnant, puisque la paix est définie dans la plupart des dictionnaires par la négative, comme une non-guerre. La situation n'est pas nouvelle, et déjà il y a plus d'un demi-siècle G. Bouthoul, qui fut pourtant l'un des plus grands spécialistes de la sociologie de la guerre, reconnaissait qu'il y en avait autant de définitions que d'auteurs s'étant penchés sur la question, et qu'il était difficile, voire impossible, de s'accorder sur une qui soit unique et figée (Bouthoul 1951 - p. 25 sq., 1962 - p. 31 sq.). Depuis, si le nombre de travaux a augmenté exponentiellement, rien n'a fondamentalement changé. Il y a plusieurs raisons à cela. L'existence de spécificités selon les approches et les disciplines en est une, mais, surtout, la guerre se présente sous une telle diversité de formes, dont les limites sont parfois très floues, que l'on ne peut en avoir la même vision selon les caractéristiques que l'on retient ou l'on met en avant. En particulier, elle existe dans des sociétés aux organisations très différentes, et si tous les spécialistes s'accordent à peu près sur ce qu'est la guerre dans les États civilisés, il en va bien autrement pour les sociétés non étatiques, certaines définitions y étant d'ailleurs tout simplement non opérationnelles.

Ainsi, on continue aujourd'hui à débattre de l'existence ou non en Préhistoire ou en ethnologie d'un phénomène dont on n'arrive pas vraiment à dire ce qu'il est, en tout cas à le caractériser d'une façon qui soit à peu près admise par le plus grand nombre. Ce flou sémantique permet en particulier à chacun d'adopter la définition qui lui sied en fonction de ses convictions. En pratique, selon celle qui sera retenue, les conflits qui surviennent dans les sociétés non étatiques, et plus particulièrement dans les sociétés dites simples, pourront ou non être reconnus comme guerres (Allen 2014 - p. 20 ; Jones et Allen 2014 - p. 354). Le 
dernier cas peut au pire conduire à «pacifying the past by definition », pour reprendre l'expression de P. Roscoe (2017).

Bien qu'il soit difficile de s'en satisfaire, comment se sortir d'une situation qui paraît totalement sans issue? Vouloir proposer une définition de la guerre qui ferait l'unanimité est probablement illusoire, mais on peut au moins tenter de trier, dans tous les éléments qui ont été avancés pour la caractériser, ceux qui semblent les plus pertinents. II apparaît en effet que ces éléments sont de deux sortes. Certains sont génériques, c'est-à-dire qu'ils expriment un caractère que l'on peut considérer comme inhérent au concept, et il faut donc les conserver. Dans la mesure où ils sont parfois très mal cernés, il faut aussi les expliciter D'autres, au contraire, sont spécifiques et ne s'appliquent qu'à des formes particulières de guerre. Il faut donc les écarter, parce qu'ils font perdre la vision d'ensemble et, surtout, font prendre le risque de ne pas considérer comme guerre quelque chose qui en serait. C'est cette démarche qui va être adoptée au cours de toute la discussion qui suit, dont le but sera d'établir la frontière entre ce qui relève du générique et ce qui relève du spécifique. En ne gardant que la première part, on peut espérer comprendre quelle est l'essence même de la guerre. Avec la seconde part, il restera ensuite toujours éventuellement possible de la subdiviser en différentes catégories.

\section{2 | Quelques éléments de définition classiques mais non pertinents}

Avant de se pencher sur les éléments de définition qui semblent les plus pertinents, il faut commencer par en écarter quelques autres, parce qu'ils sont classiquement utilisés soit pour opposer les guerres soi-disant vraies et primitives, soit pour exclure certaines formes de violence collective intergroupe de la guerre, soit encore pour classifier la guerre en différentes catégories. Dans tous les cas, ils sont trop spécifiques ou non appropriés pour une définition générique.

\subsection{1 | Organisation de la violence}

Pour certains chercheurs, en particulier les matérialistes culturels, ce qui distinguerait la guerre, c'est que ce serait une violence organisée - un aspect qu'il faut différencier de l'organisation des groupes sociaux, qui sera abordée plus loin. Hélas, on ne sait souvent pas ce que cela signifie exactement et de quelle façon la violence serait supposée devoir être organisée. Quand la précision existe, elle fait toujours référence à l'organisation militaire (idée d'une violence organisée militairement). Il est alors question de forme du recrutement, de discipline, de tactique, de logistique, éventuellement de niveau de leadership. Tout cela renvoie implicitement à l'armée de métier et à l'État (organisation de la violence au sens wébérien), et il n'y a pas à s'étonner de ce que cet élément soit mis en avant par ceux qui considèrent que la guerre n'existerait pas dans les sociétés non étatiques, puisque sous cet aspect la violence y serait naturellement désorganisée. En réalité, il n'y a pas d'exemple de société où les gens partent se battre dans le désordre, et il y a toujours un minimum d'organisation martiale. Comment pourrait-il en être autrement? Certes, sans État il n'y a pas de conscription et sans pouvoir de commandement l'exercice de l'autorité a ses limites. Mais il n'en demeure pas moins que la moindre bande qui part en expédition a un minimum de tactique et de logistique et un leader pour coordonner l'opération, laquelle fait souvent en amont l'objet de préparatifs. Et cela vaut même pour des chasseurscueilleurs nomades, tels les Aborigènes australiens, comme l'a bien montré C. Darmangeat (2020), ou ceux du Chaco sud-américain (Mendoza 2007). Aussi, il convient de s'en tenir au point de vue de l'OMS, pour qui est organisée toute violence qui n'est pas spontanée (World Health Organization 2005 - p. 1), et écarter cet élément de définition.

\subsubsection{Motifs, motivation, finalité}

De nombreux motifs, motivations ou finalités ont été incorporés dans des définitions de la guerre (il est à peu près équivalent de s'exprimer dans les trois termes, les raisons d'agir et le résultat recherché étant toujours en grande partie confondus). Certains sont plus ou moins précis (appropriation d'un territoire et de ses ressources, acquisition de prestige, etc.), d'autres bien trop vagues pour être utiles (par exemple, imposer sa volonté à l'adversaire). Malgré leur nombre, on peut ramener les motifs invoqués à quatre catégories générales et, sur cette base (sans forcément présupposer qu'elle est la plus judicieuse), classer les conflits comme suit² :

- ceux ayant pour objectif de tirer un quelconque avantage politique,

- ceux ayant pour but d'acquérir des biens de nature économique,

- ceux sous-tendus par le désir de vengeance,

- ceux qui reposent sur une nécessité rituelle ou religieuse.

Aux derniers correspondent une grande partie des guerres dites « saintes", bien que toutes ne soient pas exemptes de motivations politiques, et les conflits ayant pour but de se procurer chez un ennemi des principes ou des substances nécessaires soit à l'identité des agents sociaux (chasse aux têtes) soit à la continuation du monde (guerre fleurie des Aztèques). Toutes ces formes de conflits n'existent pas dans toutes les sociétés, et les deux premières sont notamment absentes des sociétés simples, pour des raisons facilement compréhensibles (organisation politique minimale et absence de richesse), un élément généralement mis en avant par les colombes pour rejeter la guerre dans ces sociétés. Par contre, au sein de chacune des catégories il existe d'indiscutables (et indiscutées) guerres. Cette constatation est à elle seule suffisante pour interdire de séparer guerre et non-guerre en fonction des motifs, et pour écarter cet élément d'une caractérisation générale de la guerre. On aura à revenir sur ce point à propos de la faide.

[2] On exclura de cette classification les raisons psychologiques supposées qui, selon certains anthropologues évolutionnistes, feraient que la guerre aurait été partout et toujours une stratégie par laquelle des coalitions d'hommes coopèrent pour acquérir et défendre les ressources nécessaires à la reproduction (Glowacki et al. 2017). Cette version moderne du darwinisme social, déjà présente chez Chagnon (1968), est critiquable d'un point de vue à la fois théorique, méthodologique et ethnographique (p. ex. Lizot 1989). D'autre part, en confinant la guerre à un but ultime et en reléguant ses autres motifs au rang de moyens d'atteindre ce but, elle interdit pratiquement toute analyse sociale du phénomène. 


\subsection{3 | Ampleur du conflit ou des affrontements}

Les deux notions sont évidemment corrélées et renvoient au nombre de participants soit à la guerre en général soit à des opérations en particulier. Dans le même ordre d'idée, on trouve également des références au niveau de mobilisation ou à la létalité (sur le principe que plus grande est l'ampleur et plus élevé est le nombre de morts). Dans tous les cas, on voit d'emblée qu'il ne peut s'agir d'un élément utilisable dans l'absolu : selon la société concernée, le nombre de combattants peut aller de quelques dizaines à plusieurs millions, et il n'y a strictement aucune comparaison possible entre les guerres mondiales du $20^{e}$ siècle, la guerre du Péloponnèse ou les guerres dans des sociétés non étatiques. Bouthoul (1951 - p. 31, 1962 - p. 37) considérait déjà qu'il s'agissait d'un critère " très élastique ». Il en est de même pour le nombre de morts. La guerre des Malouines n'a fait que 904 morts, ce qui est très peu au regard des effectifs engagés (plusieurs dizaines de milliers d'hommes) et ridicule en comparaison de la démographie des deux États qui s'affrontaient, tandis qu'il existe des guerres d'extermination dans les sociétés sans État. On pourrait être tenté de juger de façon relative pour contourner l'écueil. C'est la position adoptée par A. Testart dans un travail demeuré non publié : l'ampleur doit se juger à l'aune de la démographie, du type d'organisation sociale et de l'état de la société des belligérants. Partant de ce principe, il définit la guerre comme « tout conflit armé d'une certaine ampleur, parce qu'il implique chez chacun des belligérants une assez large communauté, compte tenu de leur démographie, de leur type d'organisation sociale et de l'état de leurs sociétés » (Testart 2009 - p. 247). Mais même ainsi, il est loin d'être toujours évident de déterminer par rapport à quelle référence on doit se placer : s'il n'y a pas de doute pour dire qu'une vendetta entre deux villages corses a une faible ampleur eu égard à la taille de la société française, par rapport à quoi juger l'ampleur dans une société divisée à la fois en unités de résidence et en diverses unités de parenté (lignages, clans, phratries...) ? Tout cela reste très subjectif et rend le critère de l'ampleur peu opératoire.

\subsection{4 | Degré d'évolution des techniques}

D'une manière générale, on ne voit pas quel lien définitionnel on pourrait établir entre guerre et degré d'évolution des techniques : il n'y a rien de comparable entre la guerre avec des drones et la guerre de tranchées, qui elle-même n'a rien de comparable avec la guerre médiévale, etc. C'est donc un aspect assez peu mis en avant. Mais il mérite discussion, parce qu'il est tout de même évoqué par certains archéologues, particulièrement en France. Selon eux, on ne devrait en toute rigueur parler de guerre, au moins en archéologie, qu'à partir du moment où un armement spécifique adapté au combat est attesté. La justification donnée est que l'on peut considérer qu'auparavant la fonction des armes (javelots, lances, arc...) pouvait se limiter aux activités cynégétiques, notamment à la chasse aux grands mammifères. L'accent est donc mis sur l'apparition des armes défensives ou peu ambiguës, comme l'épée, plus particulièrement métalliques. Un tel raisonnement comporte deux erreurs. La première est que " pouvait se limiter» ne signifie absolument pas « se limitait ». Que l'on utilise un outil générique pour une activité spécifique ne fait pas que l'activité est absente. En tenant ce genre de raisonnement, on pourrait tout autant dire qu'il n'y a pas d'agriculture sans houe au prétexte que le bâton à fouiller peut aussi être utilisé pour déterrer des tubercules sauvages. En vérité, partout en ethnohistoire on constate que certaines armes peuvent indifféremment servir à la chasse et à la guerre. Plus largement, des objets peuvent partager des fonctions d'outil et d'arme : au Rubané, l'herminette, l'outil à tout faire, était également utilisée pour se battre, et à toutes les époques la hache a servi aussi bien à abattre des arbres qu'à tuer des ennemis.

La seconde erreur est plus proprement archéologique : il peut très bien avoir existé des armes spécialisées soit qui ne nous sont pas parvenues soit que nous ne savons pas reconnaître. La première éventualité concerne toutes celles fabriquées en matériaux périssables, en premier lieu celles de nature défensive. On trouve ainsi des boucliers réalisés dans de tels matériaux partout dans le monde, y compris en Australie - où pas un seul n'a été découvert en contexte archéologique -, mais les armures et les casques sont également communs, notamment en Amérique du Nord où l'on en rencontre jusque chez les chasseurs-cueilleurs de l'aire culturelle subarctique (Jones 2004). C'est pourquoi l'apparition de la cuirasse ou du casque en bronze ne date certainement pas celle de l'armement défensif, et encore moins celle de la guerre ; elle date simplement l'application de la métallurgie de ce métal à un domaine donné. J. Leclerc et J. Tarrête (1988 p. 454) l'ont très bien résumé : «Il y aurait quelque naïveté à croire que l'apparition de la violence et celle de la guerre coïncident réellement avec la mise en place des conditions techniques qui permettent à des indices d'en parvenir jusqu'à nous. » Quant aux armes spécialisées que nous ne saurions pas reconnaître, l'exemple en est donné par l'étude des Pétrequin sur les flèches d'Irian Jaya, qui démontre qu'elles peuvent exister sous des types divers ayant des usages très différenciés, dont la guerre, mais que l'on ne peut pas identifier sans la grille de lecture fournie par les utilisateurs (Pétrequin et Pétrequin 1990). Une telle constatation a conduit J.-M. Pétillon (2008) à s'interroger sur l'usage des pointes barbelées magdaléniennes pour la guerre, un usage assez courant en ethnographie pour ce type de pointe, sans que l'on puisse démontrer qu'il était le même au Paléolithique, ou le seul possible.

\section{3 | Éléments distinctifs fondamentaux}

En langue française, comme en langue anglaise, la définition la plus générique de la guerre que donnent les dictionnaires est celle d'une situation conflictuelle entre deux ou plusieurs pays, États, groupes sociaux, voire individus ${ }^{3}$. Ce sens très général s'applique à toutes sortes de circonstances, et notamment à celles qui n'impliquent

[3] Les anglophones emploient war et warfare, deux termes qui sont souvent repris comme synonymes par les auteurs francophones et qui, il est vrai, sont régulièrement utilisés en anglais de façon interchangeable. En réalité, war désigne un conflit (armé ou non), tandis que warfare s'applique plus spécifiquement aux opérations au sein de ce conflit, notamment quand elles sont marquées par une caractéristique particulière, raison pour laquelle le terme est souvent accompagné d'un qualificatif (conventional, biological, chemical, nuclear, etc. warfare). Chez les anthropologues on note surtout une réelle tendance à employer warfare pour éviter d'employer war là où le concept de guerre fait débat 
pas une lutte armée : guerre des polices, guerre électronique, guerre économique, guerre des images, guerre idéologique, etc. Il est plutôt considéré comme un sens étendu, voire figuré dans certains cas, néanmoins il dit bien ce qu'est fondamentalement une guerre : une situation de conflit entre des groupes. Dans tous les exemples qui précèdent, le complément ou l'adjectif ne font qu'apporter une précision sur les moyens de la guerre ou sur qui elle concerne. Dans un sens à la fois plus restreint et plus spécifique, les mêmes dictionnaires indiquent que la guerre est « une lutte armée » (Le Grand Robert), « un conflit qui se vide par la voie des armes » (anciennes éditions du Dictionnaire de l'Académie française), un « rapport conflictuel qui se règle par une lutte armée » $(T L F)$, " a state of armed hostile conflict » (MerriamWebster). Ainsi, on peut dire en première approximation que dans sa définition la plus consensuelle, la guerre est un état de conflit armé intergroupe. Dans cette courte définition, il y a les trois grands éléments distinctifs fondamentaux de la guerre, qui vont être examinés tour à tour.

\subsection{1 | Conflit armé}

Ce premier point ne demande guère à être explicité. La guerre est d'abord une manifestation de violence au sens où l'OMS la définit (Rutherford et al. 2007), plus particulièrement de violence physique à caractère homicide impliquant nécessairement l'utilisation d'armes. C'est-à-dire qu'il s'agit d'une manifestation d'une des formes de violence armée, elle-même plus spécialement définie de façon large comme l'utilisation de la force armée pour atteindre des objectifs et/ou des gains spécifiques (World Health Organization 2005 - p. 1). La notion de conflit armé renvoie donc simplement à une lutte impliquant l'exercice de la violence armée. Il est toutefois intéressant de souligner que c'est bien cette seule notion de "armé » qui distingue le sens restreint de guerre de son sens étendu. D'autre part, lorsque l'on parle de la guerre, on se réfère le plus souvent au sens restreint et cette notion est implicite (à l'inverse, dans le sens étendu on adjoint un qualificatif à « guerre » là où il n'y a pas de lutte armée : de deux pays qui ont un conflit commercial, on dira ainsi qu'ils se livrent à une guerre économique, mais moins facilement qu'ils sont en guerre). Néanmoins, même implicite ou sous-entendu, "armé » a la même valeur que d'autres compléments ou adjectifs, c'est-à-dire qu'il apporte une précision sur le moyen de la guerre. Autrement dit, par rapport au sens étendu, on peut voir la guerre au sens spécifique comme la forme particulière de conflit intergroupe où la lutte armée est le moyen auquel on a recours.

\subsection{2 | Intergroupe}

Le sens de conflit n'exclut pas qu'il puisse être entre individus, mais lorsque l'on parle de conflit armé, il est généralement implicite que c'est entre groupes tant il paraît évident que la guerre est un phénomène collectif. C'est son trait le plus frappant et il est incontestable : deux individus qui s'opposent ne font pas la guerre, si ce n'est dans un sens très métaphorique. La guerre est donc une forme de violence collective, c'est-à-dire par des personnes qui s'identifient comme membres d'un groupe contre un autre groupe (Zwi et al. 2002 - p. 215), laquelle s'oppose à la violence interpersonnelle, entre individus et non spécifiquement destinée à promouvoir les objectifs d'un groupe ou d'une cause (Rutherford et al. 2007). Il y a une implication à cela, peut-être un peu moins évidente, mais d'importance : la guerre est un phénomène social, en tant qu'elle est une forme comme une autre de relation intergroupe, comme peuvent l'être l'échange ou la coopération. La parenté entre ces différents modes de relation sociale a d'ailleurs été soulignée par de nombreux anthropologues (Mauss 1923-1924 ; Lévi-Strauss 1967 p. 78 ; Sahlins 1976 ; Lemonnier 1990 ; Descola et Izard 1991; etc.).

Si les points précédents vont presque dans dire, le qualificatif intergroupe en soulève un autre à la fois clé et probablement le plus épineux de la définition de la guerre (et de son articulation avec la faide, comme on le verra plus loin) : toutes les luttes armées entre deux groupes quels qu'ils soient sont-elles des guerres? Sinon, lesquelles en sont et lesquelles n'en sont pas, et surtout pourquoi ? Pour poser le problème plus explicitement, soit l'on considère que des conflits peuvent être qualifiés de guerres quelle que soit la nature des groupes qu'ils opposent soit on s'y refuse, ce qui est l'opinion quasi unanime. Mais dans ce cas, il faut bien placer un seuil quelque part. Au-dessus de ce seuil, les conflits seront considérés comme intergroupes et donc comme de la guerre. Au-dessous, on leur refusera cette appellation au prétexte qu'ils sont certes collectifs, mais par la force des choses intragroupes. Cela signifie explicitement que l'on estime que les groupes impliqués ne sont alors que des sous-ensembles d'une même unité supérieure qui sert de référence. Là-dessus vient se greffer une difficulté : il y a dans toutes les sociétés de multiples façons de former des groupes (quel que soit le nom qu'on leur donne, groupes, unités, collectivités, communautés, cela revient au même, c'est entre de telles entités qu'est censée se faire la guerre). Les regroupements de personnes peuvent se faire en fonction de nombreux paramètres, les plus classiquement utilisés en anthropologie sociale renvoyant à la parenté (famille, lignage, clan...), aux niveaux sociopolitiques utilisés par les néo-évolutionnistes (bande, tribu, chefferie, état) ou à l'occupation d'un territoire commun (village, unités territoriales diverses). En outre, les membres d'une même société s'organisent simultanément en différentes sortes de groupes en fonction de ces paramètres, d'autres critères, ou au gré d'alliances diverses. Par exemple, un même village peut rassembler des membres de plusieurs lignages et, à l'inverse, un même lignage peut avoir des branches dans des villages différents. Où, comment et sur quelle base placer le seuil ?

La plupart des définitions qui distinguent l'intergroupe de l'intragroupe s'appuient sur le niveau d'organisation politique et sont des variantes de la définition princeps de Malinowski (1941 - p. 523), qui voyait « la guerre comme une lutte armée entre deux unités politiques indépendantes ». "Unités politiques indépendantes » est remplacée selon les cas par "entités souveraines », " groupes autonomes », etc., mais c'est toujours avec la même idée de deux forces qui s'affrontent sans qu'il y ait une force supérieure au-dessus. Cette référence au politique pose d'importants problèmes:

1/ D'abord, on l'a vu, tous les chercheurs ne sont pas d'accord sur le niveau politique nécessaire pour définir la guerre, certains, notamment parmi les évolutionnistes, 
ayant considéré qu'il ne pouvait y avoir de guerre autrement qu'entre États, d'autres admettant qu'elle peut exister entre unités politiques non étatiques.

2/ Quand ce sont des États qui sont en conflit, il n'y a évidemment pas de difficulté à bien cerner les contours des groupes. Et comme par nature il n'y a rien au-dessus de l'État, un conflit entre États est nécessairement intergroupe. Mais pour des sociétés non étatiques, l'absence de gouvernement central et les différences de niveaux d'organisation sociale rendent plus ou moins floue la différence intragroupe/intergroupe. Pour celles qui possèdent une forme de pouvoir politique organisé, on peut encore opérer une distinction, au prix parfois de quelques contorsions (par exemple pour la NouvelleGuinée, voir Berndt 1964 ou Roscoe 2014). Cependant, dans d'autres cas le critère politique est tout bonnement inopérant, soit que le pouvoir politique est mal défini soit que la notion même de pouvoir politique est discutable, voire n'a pas de sens. C'est notamment vrai chez les chasseurs-cueilleurs, mais plus largement dans toutes les sociétés qui sont organisées à la fois en unités de résidence et sur la parenté et qui peuvent agir indifféremment en fonction de l'une ou de l'autre. Dans de tels cas, il est impossible de définir clairement ce qui constitue une unité politique indépendante ou un groupe souverain ou autonome, et donc ce qui est violence intragroupe par rapport à ce qui est violence intergroupe (Roscoe 2017).

3/ Même lorsque la distinction est en théorie on ne peut plus nette, c'est-à-dire dans le cas de l'État, définir la guerre comme une lutte armée entre deux unités politiques indépendantes (donc deux États) ne va pas sans conduire à des incohérences. D'abord, les choses ne sont pas toujours aussi claires, ce qui a amené à parler de «new wars » à propos des conflits où les frontières entre les différentes formes de violence sont floues (Rutherford et al. 2007). Surtout, il existe des conflits internes, donc intragroupes, auxquels il est impossible de refuser l'appellation de guerre. C'est le cas par exemple des guerres de religion, des guerres ethniques, des guerres civiles, ou même des guerres des gangs (pour lesquelles certains mettent « guerre » entre guillemets, mais qui sont majoritairement considérées comme telles et qui remplissent toutes les conditions pour pouvoir parler d'état de conflit armé intergroupe). Dans tous ces exemples, les fractions belligérantes sont des sous-groupes de sociétés étatiques, et dans certains ce ne sont même pas des unités politiques.

4/ Enfin, et ce n'est pas la moindre des réserves que l'on peut faire, personne n'a jamais apporté la moindre justification à ce que pour qu'il y ait guerre il faudrait qu'un conflit mettre aux prises des unités politiques. Pourquoi, pour quelles raisons, au nom de quel principe, des conflits armés entre deux villages ou deux groupes de parenté ne pourraient-ils pas être des formes de guerre? Pour tenter de contourner, au moins en partie, ces problèmes, certains ont bien essayé de remplacer le terme " politique » par un autre. Ainsi, S. LeBlanc parle de polities (LeBlanc et Register 2003) et définit la guerre (warfare) comme un « socially sanctioned lethal conflict between independant polities » (LeBlanc 2014 - p. 27), une définition retenue par Allen et Jones (2014) dans l'ensemble de leur ouvrage. Ce terme, qui vient d'Aristote ( $L a$ Politique, livre IV ; la politeia étant le gouvernement par les classes moyennes, une des formes de gouvernement du grand nombre), n'est guère employé en français, bien qu'il ait été repris par Rousseau dans le Contrat Social (sous la forme « politie »). Et quand il l'est, c'est avec des caractéristiques variables (Leca 2012 - p. 62-63). Pour les anglophones, polity désigne une entité politique, quelles qu'en soient la taille et la forme, ou plus largement un groupe de gens unis d'une manière quelconque. Autant dire que cela manque de précision, sans d'ailleurs complètement évacuer la notion de politique. Il n'y a là qu'un subterfuge pour tenter d'escamoter une difficulté sans la régler le moins de monde, et à tout prendre autant en rester au terme de groupe.

Des esquives comme celle-là sont surtout révélatrices de la quasi-impossibilité de résoudre le problème en l'abordant sous cet angle. À partir du moment où l'on estime que toutes les luttes armées entre deux groupes sociaux ne sont pas des guerres, on ne voit pas à quel endroit on pourrait placer le curseur pour différencier intragroupe et intergroupe, et surtout pourquoi on le placerait à un endroit et pas à un autre. On doit bien admettre que quelque seuil que l'on retienne, c'est essentiellement de façon subjective. Bien sûr, on pourrait toujours décider d'adopter une définition quelconque et l'appliquer. Mais d'abord, il faudrait que ce soit la même pour tous les chercheurs, et l'on voit bien, après quasiment un siècle de débats, que le consensus est hors de portée. Ensuite, cette définition s'écarterait nécessairement de celles qui ne restreignent pas le sens de groupe, qu'elles soient utilisées dans d'autres disciplines ou qu'elles reflètent le sens commun. Enfin, et surtout, il faudrait prouver que la distinction retenue entre différents types de groupe serait pertinente du point de vue de l'analyse anthropologique, en ce sens qu'elle distinguerait parallèlement des interactions sociales réellement différentes. Il semble donc préférable - outre que cela paraît la seule façon de s'en sortir - de conserver un sens général pour les concepts de groupe et d'intergroupe, conformément aux définitions qui ont pu en être données par les anthropologues sociaux ou les psychologues sociaux qui ont travaillé sur les relations intergroupes, notamment, comme H. Tajfel, sur les conflits.

Ainsi, on peut retenir qu'un groupe social est toute collection d'individus qui se perçoivent comme membres du même ensemble social, ont une définition commune d'eux-mêmes, fréquemment un nom qui les distingue des autres, et sont perçus par ceux de l'extérieur comme membres de ce groupe. Une communauté est plus spécifiquement un groupe social dont les membres vivent ensemble, possèdent des biens communs, ont des intérêts, un but commun. Sur cette base, il est possible de distinguer les comportements interpersonnels, qui sont des interactions entre individus déterminées par leurs rapports personnels et non affectées par les groupes auxquelles ils appartiennent respectivement, et les comportements intergroupes, interactions entre individus déterminées au contraire par leur appartenance respective aux groupes et non affectées par leurs rapports personnels (Tajfel et Turner 1979). Un conflit intergroupe est donc très logiquement tout conflit entre deux ensembles de personnes qui se perçoivent réciproquement d'abord comme différents, étrangers, mais surtout comme ennemis. Il sera d'autant plus intense que cette perception sera forte et que les individus membres des groupes se comporteront en fonction de leur appartenance et non des rapports individuels (les individus agissant non plus comme individus, mais comme 
membres du groupe). Cela rejoint complètement la position de Q. Wright, pour qui la guerre met aux prises des groupes sociaux hostiles, c'est-à-dire qui "feel conscious of their distinctiveness by maintaining attitudes of hostility toward one another » (Wright 1942 - p. 9). On précisera aussi utilement qu'il s'agit bien de groupes distincts : il ne peut pas y avoir de guerre entre un groupe et un de ses sous-groupes.

\subsection{3 | État de conflit}

Il s'agit là d'un élément qui fréquemment n'est évoqué qu'entre les lignes et dont toute l'importance n'a souvent pas été saisie, au moins en archéologie et en anthropologie. Les historiens, sociologues et juristes l'ont plus volontiers souligné, et déjà au début du $17^{e}$ siècle Grotius, reprenant Cicéron dans Le Droit de la guerre et de la paix (I, I, 2, 2), définissait la guerre comme " l'état de ceux qui tâchent de vider leurs différends par les voies de la force ». La guerre est un phénomène qui perdure dans le temps. Ce n'est pas quelque chose de ponctuel, c'est un état, une situation, une période. Entrer en guerre, être en guerre, mettre fin à une guerre, en temps de guerre (vs en temps de paix), etc., sont autant d'expressions qui traduisent cela. Cette situation est d'abord une situation conflictuelle. Plus précisément, conformément à ce qui vient d'être vu, c'est la situation dans laquelle se trouvent des groupes qui se perçoivent réciproquement comme ennemis et qui entretiennent des rapports sociaux d'hostilité les uns envers les autres. C'est ensuite une situation socialement reconnue : les relations d'hostilité ne sont pas des inimitiés personnelles de quelques-uns, mais c'est tout le groupe qui reconnaît le ou les autres groupes dans leur ensemble comme ennemis. C'est enfin une situation symétrique : les relations d'hostilité sont réciproques (autrement dit, si A est en guerre avec B, B ne peut pas être autrement qu'en guerre avec $A$ ). Ces deux derniers aspects renvoient de nouveau à Wright (ibid.), qui parlait de la guerre comme étant « a definite and mutually understood pattern of behavior $»^{4}$.

Que la guerre soit un état a deux corrélats importants. Le premier est qu'il faut bien distinguer cet état des actions ou opérations telles que raids, razzias, batailles ou expéditions, qui sont ponctuelles. Ces opérations peuvent naturellement intervenir dans le cadre d'un conflit établi, et, sauf cas particulier, dans une guerre il y a une succession d'opérations, plus ou moins rapprochées, plus ou moins fréquentes (qui peuvent parfois être regroupées en campagnes, une notion qui renvoie à l'aspect organisationnel de la violence et qui est plus particulièrement applicable à des conflits étatiques). Une opération peut également être un mode d'entrée en guerre, si elle marque le début d'une relation d'hostilité prolongée. Mais raids, razzias ou expéditions peuvent aussi tout à fait être isolés et survenir en dehors de toute situation conflictuelle (par exemple, expédition de chasse

[4] L'analogie a parfois été faite entre la guerre, notamment « primitive », et la chasse, la première étant vue comme une sorte de chasse à l'homme. Au mieux, un rapprochement pourrait éventuellement être envisagé entre chasse et certaines formes d'opérations, mais l'on constate sans peine que la chasse n'a rien à voir avec la guerre : elle n'est pas un état et elle est totalement asymétrique. aux têtes, razzia économique par des pillards ou raid esclavagiste). Dans ces cas-là, il n'y a pas de guerre (ce que disait déjà Malinowski 1941 - p. 541).

Le second corrélat est qu'il faut également différencier guerre et violence armée. Si la première est un état, la seconde n'en est pas un. Elle n'est pas permanente et continue, mais elle s'exerce ponctuellement à l'occasion des actions et opérations. Cela revient à dire que, d'une part il y a la relation d'hostilité entre groupes, d'autre part il y a la façon dont va en pratique se traduire cette relation. D'un côté il y a le fond, de l'autre il y a la forme. Cela renvoie bien entendu à ce qui a été dit plus haut à propos du sens étendu de guerre et de l'élément « conflit armé » : la guerre est fondamentalement une situation conflictuelle entre groupes, mais qui n'implique pas une lutte armée et qui peut prendre d'autres visages, d'autres formes de règlement. Le recours à la violence armée est une conséquence possible de l'hostilité, mais ce n'est pas la seule, et, inversement, il peut y avoir violence armée, même collective, même intergroupe sans qu'il y ait guerre (cas des opérations isolées). Ce sont deux concepts qui se recoupent, mais aucun n'est inclus dans l'autre. Si la guerre, tel que le terme est communément compris, est un conflit armé intergroupe, avant tout c'est un rapport social d'hostilité qui a la particularité de se régler par la voie des armes. Autrement dit, la caractéristique fondamentale de la guerre n'est pas la violence armée, mais l'état conflictuel, la relation d'hostilité entre adversaires. C'est l'élément immuable sur lequel s'accordent toutes les acceptions.

Une dernière remarque est que même si l'état d'hostilité peut avoir un début et une fin précis, par exemple si la guerre est précédée d'une déclaration et est terminée par un traité ou un armistice, ce n'est pas indispensable. Bien des guerres sont la continuation de querelles personnelles, comme on va le voir, ou n'ont pas de fin clairement marquée. De la même façon, si certains estiment que l'état de guerre ne peut pas être un état permanent, on constate qu'entre certains groupes il existe des hostilités sans fin qui se traduisent par une violence armée endémique. C'est notamment le cas lorsqu'il s'agit de maintenir l'ordre du monde ou de perpétuer l'identité du groupe, cas général pour l'Amérique du Sud et centrale: il n'y a alors pas de paix possible.

\section{4 | La guerre et la faide}

Avant de synthétiser ce qui vient d'être dit à propos des trois éléments distinctifs fondamentaux et de le condenser dans une possible définition, il faut aborder un problème particulier, celui de la distinction entre la guerre et la faide (feud des anglophones). Il existe en effet une longue tradition dans la recherche en langue anglaise pour différencier (au moins en théorie) deux modes d'exercice collectif de la violence armée, war/warfare et feud (ou blood revenge), ce qui vient largement compliquer la discussion sur le concept de guerre (Dennen 1995 - p. 81 sq. pour une synthèse des problèmes). Remarquons qu'en France cette notion de faide est pratiquement cantonnée aux études médiévales et est quasi ignorée des archéologues et préhistoriens, à qui le débat échappe donc presque totalement. Cela conduit d'ailleurs à commencer par un point de terminologie. Lorsqu'il est traduit en français, le terme anglais feud l'est généralement par vendetta. C'est trop restrictif, la vendetta 
désignant en théorie spécifiquement la coutume corse, et ne s'appliquant guère qu'à la forme particulière de feud des régions méditerranéennes et du Proche-Orient (voir à ce propos la discussion dans Povolo 2015 - p. 199 sq.). Parfois, c'est « vengeance » qui est choisi, qui est au contraire un peu trop large et imprécis. Une solution pourrait être de conserver feud, mais puisque nous disposons d'un mot qui en est le strict équivalent, l'ancien français « faide », tombé en désuétude dans le langage courant mais encore très utilisé par les historiens du Moyen-Âge, il n'y a aucune raison de ne pas l'employer. Tout comme pour la guerre, il existe une importante littérature sur la faide/feud et plus largement les systèmes vindicatoires. Et comme pour la guerre, les travaux sont loin d'être tous en accord, et les critères de définition de la faide varient de l'un à l'autre. On peut en particulier retenir ceux de portée générale par E. A. Hoebel (1967) - pour les aspects juridiques -, J. BlackMichaud (1975) et C. Boehm (1984) en langue anglaise, et le recueil sur la vengeance dirigé par R. Verdier (1980-1984) en langue française.

\subsection{1 | Qu'est-ce qu'une faide ?}

La notion de faide/feud en anthropologie sociale et en ethnologie reprend directement celle de la faide du MoyenÂge qui, au départ dans le droit germanique puis plus largement dans le droit médiéval, était le droit de se venger d'un tort qui n'avait pas été réparé, quel qu'il soit (souvent un meurtre, mais cela pouvait être un affront quelconque). Il faut insister sur le fait qu'il s'agissait bien d'un droit, qui était imprescriptible : le droit de faide était perçu au MoyenÂge comme une voie juridique légitime qui, par opposition à la justice publique, était qualifiée de justice privée. En ce sens, c'était aussi une vengeance privée qui, comme on le verra plus bas, pouvait conduire à une guerre privée. D'autre part, si la faide était un droit, c'était aussi un devoir, une obligation morale. L'individu offensé pouvait, mais aussi se devait de répondre. Il s'agissait à proprement parler d'une obligation sociale de fait.

Ces deux caractéristiques, droit et devoir, sont tout à fait transposables à l'ensemble des sociétés sans État, ce qui justifie que l'on parle de faide/feud pour ces sociétés. D'une façon générale, la faide y repose partout sur un système de droit (généralement coutumier) de nature vindicatoire, c'est-à-dire qui définit dans quels cas on peut et doit se venger et selon quelles modalités. Elle est un système où la vengeance est partie intégrante du fonctionnement normal de ces sociétés. Dans les sociétés étatiques, les choses sont un peu différentes, puisque la vengeance privée y est naturellement hors droit. C'est le cas pour la plupart des vendettas des aires méditerranéenne et proche-orientale, une des raisons pour lesquelles l'emploi de cette appellation dans un sens général est discutable. On note aussi que c'est pour cela que la faide médiévale a fini par être interdite quand les pouvoirs royaux se sont renforcés (beaucoup de médiévistes estimant a contrario qu'il n'y avait pas vraiment d'États dans l'Europe féodale). Néanmoins, même au sein d'un État, dans la conception des sous-unités sociales impliquées, tuer quelqu'un pour se venger reste reconnu comme étant juste et légitime, et le cours de la faide est régulé par des règles comprises par chacune d'entre elles (Boehm 1984 - p. 218).
Au départ, la vengeance est une affaire individuelle : le principe de base est que c'est l'individu offensé qui doit répondre à son offenseur. Mais quelqu'un qui est tué ou gravement blessé ne peut évidemment pas se venger luimême. Mécaniquement, la faide suppose donc qu'une substitution du vengeur soit possible, et donc qu'il existe une solidarité entre des individus liés par le devoir de vengeance : chacun d'entre eux doit pouvoir l'accomplir au nom d'un autre. C'est pour quoi seule la vengeance qui se fait au nom de la solidarité d'un groupe est considérée comme étant de la faide, celle qui résulte d'une initiative individuelle étant écartée du concept et qualifiée par les anglophones de "vengeance killing », que l'on trouve traduit en français par « revanche ». En pratique, cette solidarité collective repose souvent sur la parenté, et elle s'exerce alors à l'échelle d'un groupe dont la taille peut être très variable (famille, lignage, clan...). Mais il peut également s'agir d'une solidarité locale, la faide étant alors menée par une communauté villageoise, une ville (cas fréquent au Moyen-Âge) ou toute une vallée. À l'inverse, fréquemment il n'est pas nécessaire que la vengeance porte spécifiquement sur le coupable, et elle peut s'exercer à l'encontre d'un autre individu appartenant à son groupe, dont tous les membres sont tenus collectivement responsables des torts subis. Ce n'est toutefois pas toujours le cas: par exemple chez les Comanche seul l'auteur d'un meurtre peut être assassiné en retour (Hoebel 1967 - p. 139). En général, la vengeance est alors limitée (voir plus bas).

Enfin, et ce n'est pas le point le moins important, pour le Moyen-Âge tous les dictionnaires de langues anciennes, anglais et allemand comme français, indiquent que « faide » désigne à la fois le processus juridique, c'est-àdire le droit de se venger, et l'état d'inimitié qui en résulte (étymologiquement faide dérive d'ailleurs du vieux hautallemand fehida qui signifie tout simplement « ennemi »). C'est donc tout naturellement que les médiévistes estiment que la faide est avant tout un état d'animosité qui se traduit par une chaîne d'actions de revanche (Povolo 2015 - p. 202). Cet avis est très généralement partagé pour les autres sociétés, et la plupart des auteurs considèrent qu'une seule vengeance n'est pas de la faide. Par exemple, à propos des Comanche évoqués plus haut, Hoebel (1967 - p. 139) précise bien que «blood-revenge killing was only of the first killer and retaliation did not lead to feud », ou pour Pospisil (1968) il faut au minimum trois actes de violence pour pouvoir parler de faide («the essence of feud has been found to be a series (at least three instances) of acts of violence »). Une simple vengeance où le contre-meurtre équilibre les comptes et éteint la rancune n'est donc pas une faide. Seul peut être considéré comme telle un système où la vengeance est elle-même une offense qui ouvre droit à vengeance, ce qui conduit à un déséquilibre et à une relation d'animosité réciproque prolongés, durant parfois des décennies. La faide est une situation conflictuelle qui se prolonge Black-Michaud (1975 - p. 16) estimant même que la faide est interminable, contrairement à la guerre, ce qui est une position extrême.

Si l'on récapitule, une faide est ainsi, en première approche, une situation de conflit qui oppose deux groupes sociaux dans le cadre d'un système vindicatoire 
et qui se traduit par une suite d'actions armées qui ont pour but de se venger d'un tort. Si d'autres critères de définition ont parfois été discutés, ils sont trop restrictifs et ne caractérisent qu'une forme particulière de faide (vendetta ou autre).

\subsection{2 | Articulation entre guerre et faide}

On voit que guerre et faide ont beaucoup en commun. Avant tout, les deux sont des états conflictuels: une opération isolée n'est pas de la guerre, et une seule vengeance n'est pas de la faide. Les deux impliquent le recours à la violence armée, sous la forme d'une suite d'actions. Les deux mettent en jeu des groupes sociaux: il n'y a pas de guerre entre individus, comme il n'y a pas de faide entre individus. Aussi, si l'on estime que ce sont deux phénomènes différents, il ne semble a priori y avoir que deux façons possibles de les distinguer:

- à la différence de la faide, la guerre ne s'inscrirait pas dans un système vindicatoire (dit autrement, il n'existerait pas de guerre motivée par la vengeance):

- ce ne seraient pas les mêmes groupes sociaux qui feraient la faide et la guerre.

La seconde option renvoie essentiellement au politique. En effet, ceux qui considèrent que la guerre ne peut être qu'un affrontement entre unités politiques estiment que, au contraire, la faide se fait en leur sein. C'était déjà la position de Malinowski (1941), pour des raisons antérieurement exposées : la faide n'est pas de la guerre parce qu'elle ne se fait pas entre unités politiques indépendantes (il parle toutefois de « intertribal feud»). Mais cette position est partagée par bien d'autres, par exemple par Pospisil (1968), qui évoque «two groups related to each other by superimposed political-structural features » et précise que " whereas feuds can occur only within a politically organized whole, war occurs beyond such an organization and always involves two groups that are politically unrelated », ou en France par Descola et Izard (1991 - p. 313). On ne s'étonnera pas que cette référence au politique pose exactement les mêmes problèmes que pour la définition de la guerre. Pour des sociétés étatiques, on n'a aucun mal à distinguer faide et guerre, ne serait-ce déjà que parce que la première relève de l'illégal. Pour des sociétés sans État, même lorsqu'il existe une forme d'organisation en matière de pouvoir politique c'est déjà plus délicat. Il faut parfois s'arranger avec le sens des termes, voire en introduire de nouveaux. Par exemple, Pospisil (1968), en plus de la faide et de la guerre, distingue un conflit qui met aux prises deux segments appartenant à deux unités politiques différentes, qu'il appelle « external self-redress »(il reprend en fait la notion de "external retaliation » de Hobhouse et al. 1915 - p. 228). Mais dans les sociétés où il n'existe pas d'unité politique formelle, comment différencier? L'option retenue est généralement de parler de faide et d'écarter la guerre au prétexte que le conflit est entre groupes de parenté, mais pas entre communautés (Allen 2014 - p. 20), une option finalement logique pour ceux qui considèrent que la guerre n'apparaît qu'à un certain niveau d'évolution des sociétés, mais qui n'en est pas pour autant moins arbitraire qu'une autre. Mais que l'on se réfère à l'existence ou non d'un système vindicatoire ou à la notion de groupe, en pratique la frontière entre guerre et faide est réellement très ténue. considérons d'abord le cas de la faide médiévale.
Unanimement, tous ceux qui en sont spécialistes ne voient aucune séparation entre la faide et la guerre. On glisse de la vengeance privée à ce qui est classiquement appelé la « guerre privée » (par opposition à celle qui est publique car menée par l'État), sans que l'on puisse distinguer la moindre limite entre elles. La seconde n'est qu'un prolongement de la première, mais les deux s'inscrivent dans un système vindicatoire et ce sont les mêmes groupes qui mènent l'une et l'autre. Plus encore, les gens du Moyen-Âge eux-mêmes ne faisaient souvent pas la différence : A. Musin (2015 - p. 10) indique que les cycles vindicatoires dont dérivent les actes de vengeance sont qualifiés de « guerre » dans les sources mêmes, et P. Monnet (2008 - p. 187) précise qu'en allemand la distinction sémantique entre krieg et fehde est tardive (15e siècle), auparavant la langue des documents ne distinguant pas les deux termes (l'opposition se faisant entre paix et guerre, quelle que soit la forme de la guerre). En ce qui concerne les sociétés sans État, de nombreux auteurs regroupent également guerre et faide, estimant qu'il n'y a pas de différence réelle entre les deux, que ce n'est au mieux qu'une question d'ampleur, et qu'on ne peut les distinguer. Par exemple, côté francophone Verdier (1980 - p. 24-25) indique que la guerre peut souvent se confondre avec la vendetta, ou côté anglophone R. C. Kelly (2000 - p. 5, 163-164) subsume war et feud sous le même terme de war (pour lui la distinction se fait entre d'un côté la revanche, assimilée à une peine capitale, et de l'autre faide et guerre). C'était déjà l'opinion de Hobhouse et al. (1915) à l'issue de leur étude de plusieurs centaines de sociétés comprenant des chasseurs-cueilleurs et des agropasteurs classés en différentes catégories: aucune distinction ne pouvait être faite entre guerre et faide, et la seule opposition qui pouvait être établie était entre d'une part les deux regroupés et d'autre part non-guerre. Mais le plus significatif est là encore que les sociétés concernées elles-mêmes conçoivent ainsi les choses. Ainsi, chez les Maenge de Nouvelle-Guinée « il est très difficile, voire impossible de distinguer [...] entre guerre et vendetta sur le plan conceptuel » (Panoff 1980 - p. 149, 1985 - p. 92). Toujours pour la Nouvelle-Guinée, Berndt (1964 - p. 192) fait le même constat à propos d'autres groupes, soulignant au passage toute la difficulté de parler d'unité politique: "Where loyalties fluctuated with residence, it is not easy to speak of a political unit, particularly since warfare necessarily involved allies. Two local descent groups might feud, with limited fighting; but as soon as either received aid from others, this was warfare. » Kroeber (1925 - p. 49) conclut de même à propos des Yurok: "No distinction of principle existed in the native mind between murder and war. It is rather clear that all so-called wars were only feuds that happened to involve large groups of kinsmen, several such groups, or unrelated fellow townsmen of the original participants. » Leach (1964 - p. 185), encore, précise en parlant des Kachin: "When Kachins talk of war (majan), they mean nothing more than a blood feud (bunglat hka) in which the lineages or two chiefs are involved and which on that account is difficult to bring to any immediate settlement. » On pourrait multiplier les exemples, on trouverait partout la même chose.

Il y a deux leçons à tirer de toutes ces données. La première, c'est que la frontière entre la faide et la guerre n'est d'évidence pas marquée par le principe de réparation. Il existe des guerres vindicatoires et, en réalité, 
dans les sociétés non étatiques se venger est un motif de guerre on ne peut plus habituel. Rappelons qu'en d'autres temps la guerre de Troie, dont nul n'a jamais douté qu'elle était une guerre entre Achéens et Troyens, n'a été motivée que par la vengeance de Ménélas contre Pâris suite au rapt d'Hélène. Du point de vue de ce seul critère, la faide ne serait donc qu'une sorte de guerre dont les motivations se situeraient dans l'orbite de la vengeance.

La seconde leçon, c'est que dans les sociétés non étatiques il est d'abord extrêmement difficile de dire que les guerres seraient externes aux groupes tandis que les faides seraient internes. Il est ensuite pratiquement impossible de dire que la faide serait le propre de certains groupes sociaux, tandis que la guerre concernerait des groupes différents (un point qui vaut également pour la faide médiévale). Ce qui ressort d'une manière générale, c'est que l'on passe insensiblement de la faide à la guerre par extension du conflit à un plus grand nombre de personnes impliquées; il y aurait une différence d'ampleur entre l'une et l'autre. Une faide serait un genre de petite guerre, et une guerre serait une faide élargie. Mais d'une part, entre les deux il n'y a pas forcément de changement de nature des groupes qui s'affrontent: on peut par exemple juste passer de groupes de parenté à d'autres plus larges, d'un conflit entre familles à un conflit entre lignages. D'autre part la nature des groupes peut changer sans que l'on puisse établir une hiérarchie entre eux, par exemple lorsqu'une querelle entre deux familles s'étend à deux villages. On retombe sur un problème déjà évoqué : où placer le curseur et, surtout, sur la base de quels critères ? Pourquoi un conflit entre familles serait-il de la faide, tandis qu'un conflit entre lignages ou villages serait de la guerre? La réponse est d'autant plus délicate que le premier est souvent assimilé à une guerre entre familles, par exemple en ethnologie par Cariage (1980), ou même, au sein d'un État, dans le cas de la « guerre des Castellammarese $»$.

Finalement, l'on constate qu'en dépit de tous les efforts classificatoires qui ont été faits pendant des décennies, distinguer guerre et faide et déterminer comment les deux s'articulent s'appuie toujours sur des critères essentiellement subjectifs. Certes, on parvient parfois à établir une limite, certaines fois sur la base des découpages en groupes propres à une société en particulier, dans d'autres sur l'ampleur du conflit, dans d'autres encore sur la façon de traiter les morts - par exemple, il semble assez général qu'on ne prend des trophées, ou tout du moins qu'on ne coupe des têtes, que dans la guerre, pas dans la faide (Karsten 1923 - p. 13-14; Boehm 1984 - p. 191). Mais c'est toujours au cas par cas et aucun critère général applicable à toutes les sociétés ne se dégage.

Si l'on essaye d'avoir une approche systématique, il n'y a au départ que deux façons possibles de considérer les choses: soit les faides sont par nature des sortes de guerres soit elles n'en sont pas mais peuvent se transformer en guerres. La première option revient à mettre en avant le seul caractère vindicatoire et à faire de la faide un sous-ensemble de la guerre : elle serait la forme vindicatoire de la guerre. Cette option possède au moins deux grands défauts. D'abord, il faudrait avoir une conception très large de la lutte armée pour inclure dans la guerre des meurtres et contre-meurtres commis par des individus isolés au nom de la solidarité collective, situation qui se rencontre régulièrement. Surtout, ce n'est pas ce que disent les observateurs: dans beaucoup de cas, il y a des faides qui ne sont pas considérées comme de la guerre, c'est seulement que l'on peut glisser de l'une à l'autre. Il faut donc plutôt envisager que les faides peuvent se transformer en guerre. Mais en quoi consiste cette transformation, étant entendu qu'elle ne peut concerner ni le caractère vindicatoire du conflit ni la nature des groupes qui s'opposent? Ce qui est le plus souvent mis en avant, c'est que le passage se traduit par une extension du conflit, avec implication d'un nombre plus grand de personnes, c'est-à-dire également par l'extension des solidarités parentales, de voisinage, ou autres. C'est naturellement vrai, mais c'est sans doute seulement la traduction d'un autre phénomène : ce qui change, ce n'est pas tant l'ampleur de la mobilisation que l'ampleur de la réponse que l'on souhaite apporter, la première ne paraissant être que la conséquence de la seconde.

Il existe une idée absolument centrale dans toutes les faides, au moins quand elles débutent, c'est celle de " parité dans la vengeance », pour reprendre l'expression de Chelhod (1980 - p. 132). Cela signifie que le groupe en tort doit subir une perte équivalente à celle qu'il a causée, « un acte violent regardé comme équivalent » (Panoff 1980 - p. 144), ce que traduit très exactement la loi du talion. Il peut s'agir d'une équivalence en quantité, par exemple quand, chez les Shuar, «for one life which has been taken only one life should be taken in retaliation» (Karsten 1923 - p. 13), ou comme on l'a vu plus haut chez les Comanche. Mais elle peut également être en qualité là où l'on estime que tous les hommes ne se valent pas et qu'il faut parfois en tuer plusieurs pour compenser la mort d'un seul (Chelhod ibid.). Soulignons que ce qui compte en pratique, ce n'est pas par qui la vengeance est exercée, mais bien contre qui elle s'exerce : un commando de plusieurs hommes peut très bien être envoyé pour tuer un seul individu. Le point essentiel, toutefois, c'est que ce mécanisme de parité est consubstantiel à la faide, parce que ce sont les règles des faides qui garantissent l'équilibre et que partout ces règles existent, puisque partout ce sont elles qui définissent en droit les façons de s'acquitter de la vengeance et les cibles jugées convenables, ce qu'il est autorisé de faire et ce qui ne l'est pas.

Mais, bien sûr, il n'y a parité... que tant que les règles sont respectées. Et, autant que l'on puisse en juger à partir des données ethnohistoriques disponibles, partout aussi il semble que c'est lorsque l'on s'affranchit des règles de la faide que l'on bascule dans la guerre. Cela ne signifie pas qu'il n'y a plus de règles du tout : la guerre est une affaire universellement réglée au moins au sein de chaque société, et il peut même y avoir des règles partagées par des groupes en guerre, y compris en l'absence de droit international moderne. C'est simplement que celles de la faide qui limitent l'ampleur de la réponse ne s'appliquent plus. On sort du droit de la faide. Dans la guerre, vindicatoire ou non, l'idée de parité n'existe plus. S'y substitue celle d'infliger le plus de dégâts possible. Quand dans la faide on recherchait l'équilibre, dans la guerre on cherche la victoire, c'est-à-dire à dominer. Et dans le cadre particulier de la vengeance, la faide est la vengeance équilibrée, mesurée, tandis que la guerre vindicatoire est la vengeance sans mesure ni recherche de l'équilibre. C'est avec ce changement d'idée que l'on passe de la première à la seconde. On n'est pas très loin d'une autre distinction, sous une certaine forme, celle entre réparation et punition 
(Radica 2018) : d'un côté on cherche plutôt à réparer à la mesure du tort originel, de l'autre à punir sans aucune mesure. Ce processus de transition d'une forme à une autre est extrêmement bien illustré par Charachidze (1980 - p. 87) dans son étude sur la vendetta chez les Abkhazes, qui en outre souligne la perte de la référence au droit: «... les "proportions grandioses" que prenait la vendetta lui retiraient en fait les caractères d'un mécanisme de régulation juridique. Pratiquée ainsi à grande échelle, la vengeance du sang se transforme en guerre de seigneuries et perd sa raison d'être, la commutation du préjudice. La notion même de contre-meurtre se dilue dans cette violence généralisée et sans frein. »

Cet exemple des Abkhazes permet d'introduire deux remarques complémentaires. Comme dans maints autres endroits du monde, il existait chez eux des mécanismes compensatoires pour mettre fin à des conflits, même ceux à grande échelle. De tels mécanismes ne sont absolument pas propres à la faide et ne la caractérisent pas, ils existent également pour la guerre, et il faut bien les différencier de la volonté de mener ou non des représailles équilibrées. D'autre part, la vengeance n'est parfois qu'un simple prétexte pour aller combattre un ennemi que l'on souhaite dominer ou dont on veut se débarrasser, et elle peut donc conduire directement à la guerre sans passer par la faide.

\section{5 | Propositions en forme de synthèse}

Pour synthétiser les longs développements qui précèdent, on peut essayer de les condenser sous la forme de définitions de la guerre et de la faide qui reprennent les éléments essentiels. Ainsi, la guerre correspond au phénomène suivant:

État conflictuel entre deux ensembles distincts de personnes (groupes) qui se perçoivent globalement et réciproquement comme ennemis et entretiennent un rapport social d'hostilité, chaque groupe tentant d'établir sa supériorité sur l'autre par le moyen de la lutte armée.

Sans doute cette définition, finalement très classique dans le sens où elle rejoint celles de Grotius ou des anciens dictionnaires, ne peut-elle pas faire consensus (mais en est-il une qui le peut?), et elle n'a de toute façon pas la prétention de définitivement clore des débats vieux d'un siècle. Au regard de ce qui a été exposé, c'est simplement une proposition qui semble pertinente pour pouvoir aborder de la façon la plus objective possible le problème de la guerre préhistorique, cela pour les raisons suivantes: - elle s'appuie sur des éléments à la fois nécessaires (tous semblent devoir être présents) et suffisants (au sens où vouloir définir la guerre par d'autres éléments reviendrait à définir une forme de guerre particulière);

- de fait, elle est assez générale pour pouvoir s'appliquer à tous les cas de figure et permettre ensuite un classement en fonction des motivations ou du niveau social des groupes impliqués (guerres entre familles, entre villages, entre lignages, entre clans, entre États...) ;

- elle fait donc de la guerre préhistorique une guerre qui conceptuellement n'est pas différente des autres (elle n'est préhistorique qu'en tant qu'elle concerne des sociétés avant l'Histoire).

Par ailleurs, si cette définition s'applique à la guerre au sens spécifique, ses deux parties distinguent l'acception générale et ce qui fait la spécificité (autrement dit le moyen), le recours à la violence armée. Ainsi :
- elle met l'accent sur la caractéristique fondamentale de la guerre, celle d'être un état ;

- elle pointe également la dimension sociale de la guerre: c'est un rapport social d'hostilité entre groupes :

- elle s'affranchit des problèmes que posent d'autres définitions, notamment de celui de déterminer ce qui est intergroupe et ce qui ne l'est pas ou ce qui est unité politique et ce qui ne l'est pas.

Cette définition inclut les conflits divers au sein des États, conflits ethniques, politiques ou religieux, guerre des gangs, guerre vindicatoire, etc. Par contre, elle exclut :

- toutes les opérations isolées qui ne s'inscrivent pas dans une situation prolongée d'hostilité ;

- tous les conflits et violences interpersonnels;

- toutes les situations qui opposent un groupe avec un de ses sous-groupes (conflit social, révolte, rébellion, répression, etc.), puisque ces groupes ne sont pas distincts; - les faides caractérisées par une réponse mesurée (voir ci-dessous).

En ce qui concerne la faide, on peut faire la proposition suivante:

La faide est une situation conflictuelle qui oppose deux groupes sociaux dont l'un au moins entretient le désir de se venger d'un tort, réel ou supposé, infligé par un ou plusieurs membres de l'autre, et qui emploie la violence armée comme moyen d'accomplir ce désir. On peut en distinguer deux formes:

- celle qui s'inscrit dans le cadre d'un système vindicatoire, c'est-à-dire un système qui définit les règles selon lesquelles on peut et doit se venger, et donne lieu à une suite de meurtres et contre-meurtres visant à chaque fois à établir la parité dans la vengeance (réponse mesurée);

- celle qui échappe à un tel système et où la lutte armée n'est plus utilisée pour exercer une réponse mesurée, mais pour se venger en imposant sa supériorité à l'autre groupe; cette forme est assimilable à de la guerre et peut être qualifiée de guerre vindicatoire.

Cette proposition essaye de traduire au mieux la réalité des faits tels que l'on peut les constater dans différents types de sociétés, tout en restant en conformité avec le concept originel. Mais l'on voit bien le problème que pose le terme de faide, dû à ce qu'il renvoie à la vengeance de façon large, problème de sémantique plus que réellement de fond. On peut en effet envisager les choses de deux façons différentes. La première, en voyant faide et guerre comme deux ensembles qui se recoupent, avec la guerre vindicatoire à leur intersection, considérée à la fois comme forme de guerre et forme de faide. On ne peut nier que cela a un côté pratique, chacun pouvant appeler un même phénomène guerre ou faide selon ce qu'il désire montrer. En particulier, ceux qui considèrent que la guerre n'apparaît qu'à un certain niveau d'évolution des sociétés peuvent avancer qu'en deçà de ce niveau les groupes ne font pas de la guerre, puisqu'ils font de la faide. La seconde façon d'envisager les choses, c'est de voir guerre et faide comme deux ensembles différents et disjoints, la guerre vindicatoire n'étant plus une sorte de faide, mais dérivant de la faide. Très probablement, cette manière de voir est plus conforme à la réalité : on peut toujours donner le nom de faide à la seconde forme définie ci-dessus, mais il n'en reste pas moins qu'elle répond pleinement à la définition de la guerre et qu'elle n'est pas autre chose que de la guerre, même dans la conception de ceux qui sont concernés. En toute logique, il serait donc 
bien préférable de restreindre le terme de faide à la seule première forme de la définition. Mais en pratique ce n'est pas ce que font les chercheurs.

\section{3 | COMMENT ABORDER LA QUESTION DE LA GUERRE PRÉHISTORIQUE ?}

Maintenant que, sans prétendre l'avoir réglé définitivement, nous avons au moins examiné le problème de sémantique de façon à nous munir d'une définition de la guerre, nous pouvons revenir sur celui de la doctrine, dont les bases ont déjà été exposées au début de cette contribution. Pour ce faire, on peut décomposer l'approche en deux étapes, qui renvoient aux deux types d'arguments avancés par ceux qui rejettent l'existence de la guerre au Paléolithique : 1) il y a des raisons pour qu'elle n'existe pas, 2) il n'y en a pas de preuve archéologique.

\section{1 | Les chasseurs-cueilleurs du Paléolithique ont-ils pu faire la guerre?}

Les arguments donnés par les colombes pour expliquer l'absence de guerres au Paléolithique se trouvent par exemple en France sous la plume de Patou-Mathis (2016 p. 28-30):

- une faible démographie,

- un territoire de subsistance suffisamment riche et diversifié,

- l'absence de biens à piller (richesses ou stocks),

- une société égalitaire, peu hiérarchisée,

- l'absence de sacrifices humains à une divinité.

Ce sont peu ou prou les mêmes que l'on retrouve de l'autre côté de l'Atlantique, par exemple, en miroir, dans les facteurs d'apparition de la guerre donnés par Ferguson (2018): "The preconditions that make war more likely include a shift to a more sedentary existence, a growing regional population, a concentration of valuable resources such as livestock, increasing social complexity and hierarchy, trade in high-value goods, and the establishment of group boundaries and collective identities. "

On ne saurait s'en étonner, tout cela renvoie essentiellement au quatuor sédentarité, stockage, richesse et inégalités, donc à la complexité sociale, agrémentée de notions plus curieuses, comme l'identité collective chez Ferguson (il n'existerait pas d'identité collective chez les chasseurs-cueilleurs égalitaires ?), où l'absence de sacrifices humains chez Patou-Mathis (dont on saisit mal le rapport avec l'absence de guerre). Quant à la faible démographie, évoquée dans les deux cas, elle laisse songeur si l'on considère que les peuples amazoniens sont parmi les plus belliqueux du monde alors qu'ils vivent dans une des régions les moins densément peuplées. Il est également important de souligner que le raisonnement suppose que les chasseurs-cueilleurs du Paléolithique étaient simples et égalitaires, ce qui n'est tout de même pas une certitude - et quoi qu'il en soit n'est pas démontré. L'hypothèse sera toutefois conservée ici : elle fait l'objet d'un autre débat, et de toute manière la question de l'existence de la guerre chez les chasseurscueilleurs complexes ne se pose pas.

Finalement, si l'on conserve le cœur de l'argumentation, l'idée est que les motifs de la guerre seraient exclusivement d'ordre économique ou politique - avec en arrière-plan celle, fondamentalement rousseauiste, que la guerre naîtrait avec la propriété -, et que, par la force des choses, sans ces motifs il ne pouvait y avoir de guerres au Paléolithique. Il faut absolument chasser cette idée, qui constitue un biais interprétatif majeur. Certes, les chasseurs-cueilleurs simples ne font pas la guerre pour des motifs économiques ou politiques, pour des raisons évidentes. Mais, ils ont deux autres grandes motivations pour la faire, déjà évoquées: punir des ennemis, notamment pour se venger, et se procurer chez eux des principes ou des substances nécessaires à l'identité des agents sociaux ou à la continuation du monde.

Ce dernier point est testable et vérifiable, et il suffit pour cela de se tourner vers les chasseurs-cueilleurs du passé récent. Font-ils la guerre? On peut d'emblée donner la réponse, telle qu'elle a été résumée par R. L. Kelly (2013b p. 158) : « it is not useful to ask whether hunter-gatherers (inclusive of egalitarian and nonegalitarian types) are peaceful or warlike; we find evidence for both among them ». Il est évident que tous les chasseurs-cueilleurs ne font pas la guerre, mais en réalité personne n'a jamais contesté ce fait. Sans qu'il y ait une corrélation stricte, on peut remarquer que les moins belliqueux sont souvent ceux qui vivent dans des milieux difficiles (Inuit, Aborigènes du désert central australien) ou qui sont enclavés et entourés par des sociétés bien plus puissantes (San). Mais il est tout aussi évident que certains chasseurs-cueilleurs, y compris égalitaires, font la guerre au sens donné ici, c'est-à-dire entretiennent des relations sociales d'hostilité avec leurs voisins, considérés comme ennemis, contre qui ils recourent à la lutte armée. La littérature anthropologique sur la guerre fournit de nombreux exemples de relations hostiles chroniques entre groupes de chasseurs-cueilleurs, qu'elle s'appuie sur le dépouillement de bases de données ou sur les témoignages d'ethnographes ou d'observateurs anciens - qui ne tergiversent pas autant que les anthropologues pour parler de guerre - (parmi bien d'autres sources, Métraux 1963 ; Ember 1975, 1978 ; Gat 1999, 2015, 2017 ; R. C. Kelly 2000 ; Mendoza 2007 ; Wrangham et Glowacki 2012 ; Boehm 2013 ; Fry et Söderberg 2013 ; R. L. Kelly 2013b ; la majorité des contributions dans Allen et Jones 2014 ; Darmangeat 2019, 2020). On peut toujours émettre des réserves sur certains cas, moins clairement documentés, mais on ne peut ignorer la tendance générale : indéniablement il existe des chasseurs-cueilleurs égalitaires qui font la guerre. Quant aux motivations avancées, ce sont bien celles attendues, la vengeance dans beaucoup de cas, mais aussi le maintien de l'ordre du monde ou la perpétuation de l'identité du groupe, des motifs particulièrement présents en Amérique du Sud et centrale. Reste la question du contact paradox. Comme pour l'existence ou non de la guerre dans les sociétés simples, il faut adopter une position mesurée. Il serait aussi peu scientifique de nier le rôle des contacts en certains endroits, que d'affirmer que partout sans exception ce sont les contacts qui ont causé l'apparition de la guerre. Cette dernière possibilité peut paraître a priori difficile à rejeter, puisque c'est une évidence que les premiers témoignages directs ne peuvent par principe pas être antérieurs aux contacts. Tant que l'on n'a pas ouvert la boîte, on ne sait pas si le chat de Schrödinger est vivant ou mort; de même, en théorie, tant que l'on n'a pas observé les sociétés on ne peut pas savoir ce qui s'y passe. Néanmoins, à la différence des chats, les hommes 
racontent des histoires, et dans certains cas au moins la mémoire collective atteste la profondeur temporelle des conflits et leur antériorité par rapport à l'arrivée de l'homme blanc. Par exemple, en Australie (Darmangeat 2019, 2020) ou en Amérique du Sud, les rapports des premiers observateurs témoignent de l'ancienneté du phénomène. Et il existe d'ailleurs dans les deux régions des mythes qui racontent que l'on fait la guerre parce qu'il en va ainsi depuis l'origine du monde. Mais, surtout, la théorie du contact néglige totalement les motivations de la guerre dans les sociétés simples, qui sont complètement étrangères à la présence de l'homme blanc. En Amérique du Sud, le moteur de la guerre est la vengeance, vengeance qui en outre s'exerce entre des groupes apparentés, appartenant à la même culture. Il existe une relation particulière entre les groupes ennemis : ce sont des groupes partenaires de vengeance, des ennemis privilégiés, et cette inimitié perpétuelle est nécessaire au bon fonctionnement du monde, à cette pérennité du cosmos. La vengeance a un caractère nécessaire et circulaire - elle est en fait assimilable à un échange de services, à un troc - (voir Clastres 1972 pour les Guayaki ou Combès 1992 pour les Tupi-Guarani). Les Occidentaux n'ont rien à voir là-dedans. Pour l'Australie, Darmangeat (ibid.) fait le même constat, et compte tenu des motivations partout avancées, on peut sans doute généraliser sans grand risque.

Finalement, il n'y a aucune raison pour que les chasseurscueilleurs du Paléolithique n'aient pas pu faire la guerre. D’un côté, les arguments habituellement avancés pour rejeter cette thèse sont irrecevables. De l'autre, il n'est pas possible de nier l'existence de la guerre dans certaines sociétés simples du passé récent et on doit exclure que le contact avec les Occidentaux en fut partout la cause. Partant de là, on ne trouve aucune raison fondamentale, aucune explication, qui pourrait permettre de justifier, et même seulement faire soupçonner, que les choses aient été différentes pour les sociétés supposées simples du passé ancien. D'autant que les motivations de la guerre dans les sociétés simples sont à peu près universelles, en particulier le désir de vengeance. Sur quels fondements pourrait-on s'appuyer pour avancer que ce désir, présent chez les chasseurs-cueilleurs égalitaires australiens, sudaméricains ou de l'Ouest-Arctique, était totalement absent chez ceux du Paléolithique?

\section{2 | L'ont-ils faite... et peut-on le prouver ?}

N'en déplaise à Ferguson (2004 - p. 54), pas plus qu'ailleurs l'absence de preuve ne peut être tenue comme preuve d'absence en ce qui concerne la guerre paléolithique. Penser le contraire est une faute interprétative qui ignore complètement que les témoignages archéologiques qui nous parviennent ne sont que le résultat d'un vaste processus de sélection taphonomique, et non pas le reflet fidèle de la réalité du passé. Il est certes exact qu'il existe bien plus de vestiges potentiellement indicateurs d'un contexte guerrier à partir du Néolithique que pour le temps des chasseurs-cueilleurs. Mais en conclure que c'est parce que la guerre n'existait pas chez ces derniers est un raccourci fallacieux, car il existe bien d'autres raisons possibles à cette constatation, qu'il faudrait auparavant avoir toutes éliminées. En dehors des pro- blèmes généraux de conservation qui se posent sur des échelles de temps aussi longues, on peut souligner trois aspects importants :

- D'abord, ceux qui parlent de la faiblesse du nombre de cas paléolithiques de violence au regard de l'étendue géographique et de la durée de la période considérée (PatouMathis 2016 - p. 33) oublient que l'on peut en dire autant du nombre de morts. Si l'on veut pouvoir s'affranchir de l'influence des facteurs de bais, ce n'est pas à l'espace ou au temps qu'il faut rapporter le nombre de cas de violence, mais au nombre de morts connus pour la période. Les cas de violence sont bien plus nombreux au Néolithique qu'au Paléolithique, mais le nombre d'individus dont les restes nous sont parvenus est lui aussi incomparablement plus élevé. Si l'on juge proportionnellement, la vision est complètement différente (sur ce point, voir par exemple Estabrook 2014 - p. 66).

- Parmi les facteurs de biais, il en est un dont l'influence est primordiale, c'est le mode de vie. Quasiment tous les témoignages néolithiques (et postérieurs) de violence collective proviennent de structures en creux dont la plupart se trouvent dans des habitats structurés. C'est bien sûr en lien avec la sédentarité, qui explique d'ailleurs aussi l'insigne rareté des regroupements de tombes en plein air avant le Mésolithique. Mais que devenaient les victimes d'affrontements appartenant à des groupes mobiles en l'absence d'habitats en dur et de cimetières, et peut-on seulement les retrouver? Sans doute, comme le prouve le cas de Nataruk au Kenya (Mirazón Lahr et al. 2016), mais il faut un concours de circonstances exceptionnelles. Même pour le Néolithique, si l'on écarte les exemples découverts en fosses ou fossés d'habitats, il n'y a quasiment plus aucun témoignage de violence collective. Il n'est finalement pas surprenant que tous les cas paléolithiques qui méritent discussions proviennent de cavités naturelles, et pour le Paléolithique européen il est presque improbable que l'on retrouve un jour des traces de massacre dans un site de plein air.

- Enfin, peut-être ne voyons-nous pas la guerre préhistorique simplement parce que nous ne savons pas en reconnaître les traces. La remarque vaut en particulier pour le cannibalisme, maintenant documenté depuis le Paléolithique ancien. Son lien avec la guerre, ou en tout cas avec la violence armée intergroupe est universel (Guille-Escuret 2010-2013). Ce n'est pas le seul lien possible et il est archéologiquement difficile à démontrer (Boulestin 2014), mais incontestablement on aurait tort de rejeter d'emblée ce possible témoignage. La réévaluation récente des restes humains badegouliens de la grotte du Placard, en Charente, montre d'ailleurs que dans certains cas favorables on peut trouver des indices qui convergent pour faire d'activités guerrières la meilleure explication à un épisode de cannibalisme préhistorique (Boulestin et Henry-Gambier 2019).

Il y a donc bien des raisons à ce que la guerre préhistorique soit presque invisible archéologiquement. Cela rejoint tout à fait les constatations de C. Darmangeat $(2019,2020)$ à propos de l'Australie : alors que des conflits armés, parfois très sanglants, entre groupes aborigènes y sont attestés par l'ethnographie, il n'existe aucune trace archéologique de violence intergroupe.

En résumé, non seulement absence de preuve archéologique ne signifie pas absence de guerre paléolithique, mais il y a avec le cannibalisme des indices sérieux que la violence armée intergroupe existe depuis des 
temps très anciens. Pour autant, pour une autre raison que la rareté des traces, démontrer la guerre est un véritable challenge, et l'on peut même se demander si c'est tout simplement faisable. Cela tient à la définition de la guerre qui a été donnée ici. Prouver l'existence de la violence armée ne pose pas réellement de problème. Prouver que cette violence était intergroupe peut encore se faire dans des circonstances favorables, même si c'est déjà plus difficile. Mais comment prouver que cette violence s'inscrivait au sein d'un état conflictuel ? Comment démontrer une relation d'hostilité prolongée? C'est quasiment impossible, et même pour des cas comme Jebel Sahaba ou Nataruk, comment savoir qu'il ne s'est pas agi d'une razzia isolée dans le premier, ou d'un groupe massacré après avoir violé le territoire d'un autre dans le second $^{5}$ ? À vrai dire, le problème n'est pas propre au Paléolithique, et l'on peut se poser exactement les mêmes questions pour beaucoup de témoignages postérieurs. En pratique, il sera extrêmement difficile d'aller au-delà de la notion de violence armée intergroupe pour les sociétés du Paléolithique et de démontrer la guerre, mais ce n'est pas pour autant qu'il faut en écarter la possibilité, et elle doit rester au rang des hypothèses à envisager.

\section{CONCLUSION : \\ PENSER LA GUERRE PRÉHISTORIQUE}

Aborder la question de la guerre préhistorique demande d'abord de s'affranchir de la vision strictement rousseauiste ou de celle strictement hobbesienne. Il n'est pas plus raisonnable de considérer l'Homme comme un bon sauvage que comme un loup pour l'Homme, et une approche scientifique équilibrée suppose avant toute chose de se libérer des a priori et d'adopter une attitude à la fois intermédiaire et mesurée, à l'image de K. Otterbein. Examinées objectivement, les données ethnohistoriques démontrent qu'il y a toute une gamme entre des sociétés très belliqueuses et d'autres qui le sont peu. Plus encore, chaque groupe social entretient des rapports sociaux différents avec ses voisins, tantôt de coopération ou d'échange, tantôt d'hostilité donnant lieu à une lutte armée ; ce n'est ni tout l'un ni tout l'autre. De ce point de vue, les données ethnographiques montrent aussi une très forte corrélation entre la nature des rapports et la distance sociale entre les groupes : plus celle-ci augmente, plus les sentiments d'inimitié sont grands. L'étranger, celui que l'on ne connaît pas bien, que l'on ne comprend pas, est par défaut un ennemi. Sur un plan théorique, cela rejoint totalement les développements des psychologues sociaux comme Tajfel et Turner. Et l'on ne peut manquer de rappeler que la fameuse sentence "homo homini lupus est », si souvent reprise, notamment par Hobbes, l'a été sous une forme tronquée. Dans celle originelle, chez Plaute (Asinaria II, 4), la locution est « lupus est homo homini, non homo, quom qualis sit non novit », ce qui signifie en substance que l'homme que l'on ne connaît pas n'est pas un homme, c'est un loup, autrement dit une menace potentielle. Plaute ne mettait pas du tout l'accent sur la nature agressive de
l'Homme, mais sur sa peur de l'inconnu. Plus que la violence elle-même, c'est peut-être cela qui est dans la nature humaine, la crainte de l'étranger, de celui qui est différent. Au moins sous cet aspect, l'universalisme est une notion moderne et très ethnocentrique.

En ce qui concerne la Préhistoire, démontrer la guerre est un authentique défi, notamment parce que prouver un rapport social d'hostilité prolongé paraît hors de portée. Néanmoins, a contrario on ne conçoit aucune raison pour laquelle elle n'aurait pas existé : rien ne laisse penser que ce qui la sous-tend dans les sociétés du passé récent n'était pas déjà présent dans celles du passé ancien. Cela vaut en particulier pour les sociétés dites simples, étant entendu qu'il faut absolument abandonner cette idée que la guerre naîtrait de la complexification des sociétés : la complexification ne fait qu'ajouter de nouvelles motivations pour faire la guerre à d'autres déjà existantes, à commencer par le pillage des richesses. Mais d'une manière générale, il serait naïf de croire que l'inimitié est un sentiment qui est brutalement apparu à la fin des temps glaciaires. Et puis, il ne faut pas laisser la sémantique masquer l'essentiel: guerre ou non, état d'hostilité prolongé ou non, on tue rarement ses alliés ou ses amis. C'est pour quoi toutes les manifestations d'une violence que l'on peut interpréter comme intergroupe, massacres ou cas d'exocannibalisme, sont des témoignages évidents d'actes hostiles, pour le Paléolithique comme pour d'autres périodes. D'un point de vue terminologique, on parlera cependant de guerre uniquement si ces actes traduisent une hostilité persistante, et non momentanée. La différence est-elle vraiment fondamentale? Quoi qu'il en soit, si l'on ne peut archéologiquement démontrer que ces témoignages correspondent à de la guerre, au minimum il faut inclure celle-ci dans la liste des hypothèses. Cela ne signifie pas pour autant que les sociétés paléolithiques passaient toutes leur temps à se battre. Comme en ethnologie, il a probablement existé toute une gamme dans la bellicosité, allant de très à très peu, et chaque groupe devait probablement entretenir des relations différentes avec ses voisins. Et cela ne signifie pas non plus que la guerre est naturelle, inscrite dans nos gènes : fondamentalement, la guerre est l'un des comportements possibles de l'homme en société.

Enfin, il faut insister sur un dernier point : il paraît difficile de souscrire aux conclusions de Ferguson et de s'interdire d'utiliser les données ethnographiques. Au contraire, seule leur prise en compte aux côtés des données archéologiques, au sein d'une approche interdisciplinaire, est à même de générer les cadres épistémologiques nécessaires pour aider à la compréhension de la violence armée, tant dans sa dimension historique que sociologique. Dans un cas comme dans l'autre, il convient d'examiner les témoignages avec prudence et objectivité, mais seuls ceux rassemblés à partir des modes de vie des sociétés traditionnelles, ou des données historiques des populations anciennes sont susceptibles de fournir à la fois l'aperçu le plus immédiat sur la diversité des formes humaines de la violence armée et des modèles pour comprendre ce qu'ont pu être ces formes dans un passé lointain. 


\section{REMERCIEMENTS}

Je suis redevable à Christophe Darmangeat et Maurice Fhima de nombreux échanges fructueux qui ont contribué à l'avancement de ma réflexion, ainsi que de corrections et remarques sur une première version du texte. J'ai donc un très grand plaisir à les remercier ici. Bien entendu, mes propos ne les engagent nullement. Mes remerciements vont également à Jean Guilaine, pour ses commentaires sur le manuscrit initial. Enfin, je suis reconnaissant à Emmanuelle Boulestin pour la traduction du résumé.

\section{RÉFÉRENCES BIBLIOGRAPHIQUES}

ALLEN M. W. 2014 - Hunter-Gatherer Conflict: The Last Bastion of the Pacified Past? In : M. W. Allen et T. L. Jones (Eds.), Violence and Warfare among Hunter-Gatherers. Walnut Creek, Left Coast Press Inc., p. 15-25.

ALLEN M. W., JONES, T. L. (Eds.) 2014 - Violence and Warfare among Hunter-Gatherers. Walnut Creek : Left Coast Press Inc., $391 \mathrm{p}$.

BAECHLER J. 2019 - Guerre, histoire et société. Éléments de polémologie. Paris : Hermann, $460 \mathrm{p}$.

BERNDT R. M. 1964 - Warfare in the New Guinea Highlands. American Anthropologist, NS 66, 4, [Part 2: New Guinea: The Central Highlands], p. 183-203.

BLACK-MICHAUD J. 1975 - Cohesive Force: Feud in the Mediterranean and the Middle East. Oxford : Basil Blackwell, $X X X+270 p$

BOEHM C. 1984 - Blood Revenge: The Anthropology of Feuding in Montenegro and Other Tribal Societies. Lawrence : Kansas University Press, XIX + 263 p.

BOEHM C. 2013 - The Biocultural Evolution of Conflict Resolution Between Groups. In : D. P. Fry (Ed.), War, Peace, and Human Nature. The Convergence of Evolutionary and Cultural Views. New York, Oxford University Press, p. 315-340.

BOULESTIN B. 2014 - Manger son ennemi : le cannibalisme préhistorique et la «guerre ». In : O. Buchsenschutz, O. Dutour et C. Mordant (Eds.), Archéologie de la violence et de la guerre dans les sociétés pré et protohistoriques. Paris, éditions du CTHS, p. 37-52.

BOULESTIN B., HENRY-GAMBIER D. 2019 - Les restes humains badegouliens de la grotte du Placard. Cannibalisme et guerre il y a 20000 ans. Oxford Archaeopress, IX $+138 \mathrm{p}$.

BOUTHOUL G. 1951 - Traité de Sociologie. Les Guerres. Éléments de polémologie. Paris: Payot, 550 p.

BOUTHOUL G. 1962 - Le phénomène-guerre. Paris : Payot, 283 p.

CARIAGE P. 1980 - Guerre et guerre entre familles chez les Yuko. Bulletin de la Société suisse des américanistes, 44, p. 13-26.

CHAGNON N. A. 1968 - Yanomamö: The Fierce People. New York: Holt, Rinehart and Winston, XIV + 142 p.
CHARACHIDZE G. 1980 - Types de vendetta au Causasse. In : R. Verdier (Ed.), La vengeance. Volume II. Vengeance et pouvoir dans quelques sociétés extra-occidentales. Paris, éditions Cujas, p. 83-105.

CHELHOD J. 1980 - Équilibre et parité dans la vengeance du sang chez les Bédouins de Jordanie. In : R. Verdier (Ed.), La vengeance. Volume I. Vengeance et pouvoir dans quelques sociétés extra-occidentales. Paris, éditions Cujas, p. $125-143$.

CLASTRES P. 1972 - Chronique des Indiens Guayaki. Paris : Plon (collection Terre humaine), 356 p.

CLASTRES P. 1980 - Recherches d'anthropologie politique. Paris : éditions du Seuil, 247 p.

CLASTRES P. 1997 - Archéologie de la violence : la guerre dans les sociétés primitives. La Tour d'Aigues : éditions de l'Aube, $77 \mathrm{p}$.

COMBÈS I. 1992 - La tragédie cannibale chez les anciens Tupi-Guarani. Paris : Presses universitaires de France, 276 p.

DARMANGEAT C. 2019 - Vanished Wars of Australia: The Archaeological Invisibility of Aboriginal Collective Conflicts. Journal of Archaeological Method and Theory. https://doi.org/10.1007/s10816-019-09418-w.

DARMANGEAT C. 2020 - La lance de mort. Guerre et justice chez les chasseurs-cueilleurs d'Australie. Paris: EHESS, mémoire d'HDR.

DEMOULE J.-P. 2017 - Les dix millénaires oubliés qui ont fait l'histoire. Quand on inventa l'agriculture, la guerre et les chefs. Paris : Fayard, 316 p.

DENNEN J. M. G. van der 1995 - The Origin of War: The Evolution of a Male-Coalitional Reproductive Strategy. Groningen : Origin Press, 861 p.

DESCOLA P., IZARD M. 1991 - Guerre. In : P. Bonte et M. Izard (Eds.), Dictionnaire de l'ethnologie et de l'anthropologie. Paris, Presses universitaires de France, p. 313-316.

EMBER C. R. 1975 - Residential Variation among HunterGatherers. Behavior Science Research, 10, p. 199-227.

EMBER C. R. 1978 - Myths about Hunter-Gatherers. Ethnology, 17, 4, p. 439-448.

ESCALON de FONTON M. 1964 - Naissance de la guerre en Occident aux temps préhistoriques. Archéologia, 1, p. 31-34.

ESTABROOK V. H. 2014 - Violence and Warfare in the European Mesolithic and Paleolithic. In : M. W. Allen et T. L. Jones (Eds.), Violence and Warfare among HunterGatherers. Walnut Creek, Left Coast Press Inc., p. 49-69.

FEINMAN G., NEITZEL J. 1984 - Too Many Types: An Overview of Sedentary Prestate Societies in the Americas. In : M. B. Schiffer (Ed.), Advances in Archaeological Method and Theory: Volume 7. Orlando, Academic Press, Inc., p. 39-102.

FERGUSON R. B. 1992 - Tribal Warfare. Scientific American, 266, 1, p. 108-113. 
FERGUSON R. B. 2001 - Materialist, Cultural and Biological Theories on why Yanomami Make War. Anthropological Theory, 1, 1, p. 99-116.

FERGUSON R. B. 2004 - La naissance de la guerre. La Recherche, 373, p. 51-56.

FERGUSON R. B. 2008 - War Before History. In : P. de Souza (Ed.), The Ancient World at War. A Global History. London, Thames \& Hudson, p. 15-27.

FERGUSON R. B. 2018 - Why we Fight. Scientific American, 319,3, p. $76-81$.

FERGUSON R. B., FARRAGHER L. E. 1988 - The Anthropology of War. A Bibliography. New York: Harry Frank Guggenheim Foundation (Occasional Papers of the Harry Frank Guggenheim Foundation 1), XIV + 361 p.

FERGUSON R. B., WHITEHEAD N. L. (eds.) 1992 - War in the Tribal Zone. Expanding States and Indigenous Warfare. Santa Fe : School of American Research Press, XI + 303 p.

FRY D. P. 2007 - Beyond War. The Human Potential for Peace. Oxford : Oxford University Press, VIII + 331 p.

FRY D. P. (Ed.) 2013 - War, Peace, and Human Nature. The Convergence of Evolutionary and Cultural Views. Oxford: Oxford University Press, XVIII + 562 p.

FRY D. P., SÖDERBERG P. 2013 - Lethal Aggression in Mobile Forager Bands and Implications for the Origins of War. Science, 341, p. 270-273 (and supplementary materials).

GAT A. 1999 - The Pattern of Fighting in Simple, Small-Scale, Prestate Societies. Journal of Anthropological Research, 55,4 , p. 563-583.

GAT A. 2006 - War in Human Civilization. Oxford : Oxford University Press, $X V+822 p$.

GAT A. 2015 - Proving Communal Warfare Among HunterGatherers: The Quasi-Rousseauan Error. Evolutionary Anthropology, 24, p. 111-126.

GAT A. 2017 - The Causes of War and the Spread of Peace. But Will War Rebound? New-York: Oxford University Press, XIV + $303 p$.

GLOWACKI L., WILSON M. L., WRANGHAM R. W. 2017 - The Evolutionary Anthropology of War. Journal of Economic Behavior \& Organization. http://dx.doi.org/10.1016/i.jebo.2017.09.014.

GODELIER M. 1978 - Territory and Property in Primitive Society. Social Science information, 17, 3, p. 399-426.

GUILAINE J. 2011 - La violence dans la Préhistoire. In : M.-C. Marandet (Ed.), Violence(s) de la Préhistoire à nos jours. Les sources et leur interprétation. Perpignan, Presses Universitaires de Perpignan, p. 13-25.

GUILAINE J., ZAMMIT J. 2001 - Le sentier de la guerre. Visages de la violence préhistorique. Paris : éditions du Seuil, 371 p.

GUILLE-ESCURET G. 2010-2013 - Sociologie comparée du cannibalisme. Paris: Presses universitaires de France (3 volumes).
HAAS J. (ed.) 1990 - The Anthropology of War. Cambridge : Cambridge University Press, XIV + 242 p.

HASS C. 2019 - Aujourd'hui la guerre. Penser la guerre, Clausewitz, Mao, Schmitt, adm. Bush. Paris : Fayard, 399 p.

HOBHOUSE L. T., WHEELER G. C., GINSBERG M. 1915 - The Material Culture and Social Institutions of the Simpler Peoples. An Essay in Correlation. London : Chapman \& Hall Ltd., 299 p.

HOEBEL E. A. 1967 [1954] - The Law of Primitive Man. A Study in Comparative Legal Dynamics. Cambridge: Harvard University Press, VIII + 357 p.

JONES D. E. 2004 - Native North American Armor, Shields, and Fortifications. Austin : University of Texas Press, XVI + $188 \mathrm{p}$.

JONES T. L., ALLEN M. W. 2014 - The Prehistory of Violence and Warfare among Hunter-Gatherers. In : M. W. Allen et T. L. Jones (Eds.), Violence and Warfare among HunterGatherers. Walnut Creek, Left Coast Press Inc., p. 353-371.

KARSTEN R. 1923 - Blood Revenge, War, and Victory Feasts Among the Jibaro Indians of Eastern Ecuador. Washington: Government Printing Office (Smithsonian Institution, Bureau of American Ethnology, Bulletin 79), VII + 94 p.

KEELEY L. H. 1996 - War before Civilization. The Myth of the Peaceful Savage. New York: Oxford University Press, XIV + $245 \mathrm{p}$.

KELLY R. C. 2000 - Warless Societies and the Origin of War. Ann Arbor: The University of Michigan Press, X + 192 p.

KELLY R. L. 2013a - The Lifeways of Hunter-Gatherers. The Foraging Spectrum (2nd Ed). Cambridge: Cambridge University Press, $\mathrm{XIX}+362 \mathrm{p}$.

KELLY R. L. 2013b - From the Peaceful to the Warlike. Ethnographic and Archaeological Insights into HunterGatherer Warfare and Homicide. In : D. P. Fry (Ed.), War, Peace, and Human Nature. The Convergence of Evolutionary and Cultural Views. New York, Oxford University Press, p. 151-167.

KROEBER A. L. 1925 - Handbook of the Indians of California. Washington: Government Printing Office (Smithsonian Institution, Bureau of American Ethnology, Bulletin 78), XVIII + $995 p$.

LEACH E. R. 1964 - Political Systems of Highland Burma. A Study of Kachin Social Structure. London: G. Bell and Sons, Ltd., $X X+324 p$.

LEBLANC S. A. 2014 - Forager Warfare and Our Evolutionary Past. In : M. W. Allen et T. L. Jones (Eds.), Violence and Warfare among Hunter-Gatherers. Walnut Creek, Left Coast Press Inc., p. 26-46.

LEBLANC S. A., REGISTER K. E. 2003 - Constant Battles: Why we Fight. New York: St Martin's Griffin Edition, XV + 271 p.

LECA J. 2012 - L'état entre politics, policies et polity ? Ou peut-on sortir du triangle des Bermudes? Gouvernement et action publique, 1, p. 59-82. 
LECLERC J., TARRÊTE J. 1988 - Guerre. In : A. Leroi-Gourhan (Ed.), Dictionnaire de la Préhistoire. Paris, Presses universitaires de France, p. 453-454.

LEMONNIER P. 1990 - Guerres et festins. Paix, échanges et compétition dans les Highlands de Nouvelle-Guinée. Paris: éditions de la Maison des sciences de l'Homme, 187 p.

LEVI-STRAUSS C. 1967 - Les Structures élémentaires de la parenté. Paris, La Haye : Mouton, XXX + 591 p.

LIZOT J. 1989 - À propos de la guerre. Une réponse à N. A. Chagnon. Journal de la Société des américanistes, 75, p. 91-113.

MALINOWSKI B. 1941 - An Anthropological Analysis of War. The American Journal of Sociology, 46, 4, p. 521-550.

MAUSS M. 1923-1924 - Essai sur le don : forme et raison de l'échange dans les sociétés archaïques. L'Année sociologique, NS 1, p. 30-186.

MENDOZA M. 2007 - Hunter-Gatherers' Aboriginal Warfare in Western Chaco. In : R. J. Chacon et R. G. Mendoza (Eds.), Latin American Indigenous Warfare and Ritual Violence. Tucson, The University of Arizona Press, p. 198-211.

MÉTRAUX A. 1963 - Warfare, Cannibalism, and Human Trophies. In : J. H. Steward (Ed.), Handbook of South American Indians. Volume 5. The Comparative Ethnology of South American Indians. New York, Cooper Square Publishers, Inc., p. 383-409, (Smithsonian Institution, Bureau of American Ethnology Bulletin 143).

MIRAZÓN LAHR M., RIVERA F., POWER R. K., MOUNIER A., COPSEY B. et al. 2016 - Inter-Group Violence among Early Holocene Hunter-Gatherers of West Turkana, Kenya. Nature, 529, p. 394-398.

MONNET P. 2008 - La ville et la guerre dans quelques cités de l'Empire aux XIV et XVe siècles: de l'urgence immédiate à la mémoire identitaire. In : C. Raynaud (Ed.), Villes en guerre: XIVe-XVe siècles. Aix-en-Provence, Presses universitaires de Provence, p. 185-223.

MUSIN A. 2015 - Le droit de vengeance et son déclin dans les Pays-Bas (XIVe-XVIe siècles). Krypton, 5-6, p. 9-16.

OTTERBEIN K. F. 1997 - The Origins of War. Critical Review, 11,2 , p. 251-277.

OTTERBEIN K. F. 1999 - A History of Research on Warfare in Anthropology. American Anthropologist, 101, 4, p. 794-805.

OTTERBEIN K. F. 2004 - How War Began. College Station, Texas: A\&M University Press (Texas A\&M University Anthropology Series 10), XV + 292 p.

PANOFF M. 1980 - Homicide et vengeance chez les Maenge de Nouvelle-Bretagne. In : R. Verdier (Ed.), La vengeance. Volume II. Vengeance et pouvoir dans quelques sociétés extra-occidentales. Paris, éditions Cujas, p. 141-161.

PANOFF M. 1985 - La violence et la dette chez les Maenge de Nouvelle-Bretagne. Journal de la Société des océanistes, 80,41 , p. 87-100.

PATOU-MATHIS M. 2013 - Préhistoire de la violence et de la guerre. Paris : Odile Jacob, 208 p.
PATOU-MATHIS M. 2016 - La guerre a-t-elle existé au Paléolithique? In : J. Guilaine et J. Sémelin (Eds.), Violences de guerre, violences de masse. Une approche archéologique. Paris, La Découverte, p. 23-36.

PÉTILLON J.-M. 2008 - Des barbelures pour quoi faire? Réflexions préliminaires sur la fonction des pointes barbelées du Magdalénien supérieur. Palethnologie, 1, p. 69-102.

PÉTREQUIN A.-M., PÉTREQUIN P. 1990 - Flèches de chasse, flèches de guerre. Le cas des Danis d'Irian Jaya (Indonésie). Bulletin de la Société préhistorique française, 87, 10-12, p. 484-511.

POSPISIL L. 1968 - Feud. In : D. L. Sills (Ed.), International Encyclopedia of the Social Sciences. Volume 5. Springfield, Crowell Collier and Macmillan, inc., p. 389-393.

POVOLO C. 2015 - Feud and Vendetta: Customs and Trial Rites in Medieval and Modern Europe. A LegalAnthropological Approach. Acta Histriae, 23, 2, p. 195-244.

RADICA G. 2018 - Réparer et punir : trois modèles philosophiques. Rue Descartes, 93, p. 137-147.

ROSCOE P. 2014 - Foragers and War in Contact-Era New Guinea. In : M. W. Allen et T. L. Jones (Eds.), Violence and Warfare among Hunter-Gatherers. Walnut Creek, Left Coast Press Inc., p. 223-240.

ROSCOE P. 2017 - The Anthropology of War and Violence. In : Encyclopedia of Life Support Systems (EOLSS). Ethnology, Ethnography and Cultural Anthropology, Oxford, EOLSS Publishers. En ligne, http://www.eolss.net (avril 2019).

RUTHERFORD A., ZWI A. B., GROVE N. J., BUTCHART A. 2007 Violence: a glossary. Journal of Epidemiology and Community Health, 61, p. 676-680.

SAHLINS M. 1976 - Âge de pierre, âge d'abondance. L'économie des sociétés primitives. Paris : éditions Gallimard (Bibliothèque des sciences humaines), 416 p.

TAJFEL H., TURNER J. C. 1979 - An Integrative Theory of Intergroup Conflict. In : S. Worchel et W. Austin (Eds.), The Social Psychology of Intergroup Relations. Monterey, Brooks/Cole, p. 33-48.

TESTART A. 2005 - Éléments de classification des sociétés. Paris : éditions Errance, $156 \mathrm{p}$.

TESTART A. 2009 - Principes de sociologie générale. III L'État, le droit, la guerre. Tapuscrit non publié.

TURNEY-HIGH H. H. 1949 - Primitive War. Its Practice and Concepts. Columbia : University of South Carolina Press, $\mathrm{XV}+288 p$

VERDIER R. 1980 - Le système vindicatoire. Esquisse théorique. In : R. Verdier (Ed.), La vengeance. Volume I. Vengeance et pouvoir dans quelques sociétés extra-occidentales. Paris, éditions Cujas, p. 11-42.

VERDIER R. (Ed.) 1980-1984 - La vengeance. Paris : éditions Cujas (4 volumes). 
WENDORF F. 1968 - Site 117: A Nubian Final Paleolithic Graveyard near Jebel Sahaba, Sudan. In : F. Wendorf (Ed.), The Prehistory of Nubia. Dallas, Southern Methodist University, p. 954-995.

WERLY N. 2002 - Paix : l'insaisissable définition. Éla. Études de linguistique appliquées, 128, p. 481-495.

WORLD HEALTH ORGANIZATION 2005 - The Global Armed Violence Prevention Programme (AVPP). Phase I: Support for the Development of a Framework to Address the Impacts of Armed Violence on Human Security and Development. Programme Document. En ligne, https://www.who.int/violence injury prevention/violence/activities/avpp.pdf (avril 2019).
WRANGHAM R. W. 1999 - Evolution of Coalitionary Killing. Yearbook of Physical Anthropology, 110, S29, p. 1-30.

WRANGHAM R. W., GLOWACKI L. 2012 - Intergroup Aggression in Chimpanzees and War in Nomadic HunterGatherers: Evaluating the Chimpanzee Model. Human Nature, 23, 1, p. 5-29.

WRIGHT Q. 1942 - A Study of War. Chicago: The University of Chicago Press (2 volumes).

ZWI A. B., GARFIELD R., LORETTI A. 2002 - Collective violence. In : E. G. Krug, L. L. Dahlberg, J. A. Mercy, A. B. Zwi et R. Lozano (Eds.), World Report on Violence and Health. Geneva, World Health Organization, p. 213-239. 
